\title{
Investigation into the Dynamics and Control of an Underwater Vehicle-Manipulator System
}

\author{
Mohan Santhakumar \\ Centre for Robotics and Control, Mechanical Engineering, Indian Institute of Technology Indore, Madhya Pradesh 452017, India \\ Correspondence should be addressed to Mohan Santhakumar; santha_radha@yahoo.co.in
}

Received 12 July 2013; Accepted 19 September 2013

Academic Editor: Ahmed Rachid

Copyright (C) 2013 Mohan Santhakumar. This is an open access article distributed under the Creative Commons Attribution License, which permits unrestricted use, distribution, and reproduction in any medium, provided the original work is properly cited.

\begin{abstract}
This study addresses the detailed modeling and simulation of the dynamic coupling between an underwater vehicle and manipulator system. The dynamic coupling effects due to damping, restoring, and inertial effects of an underwater manipulator mounted on an autonomous underwater vehicle (AUV) are analyzed by considering the actuator and sensor characteristics. A model reference control (MRC) scheme is proposed for the underwater vehicle-manipulator system (UVMS). The effectiveness of the proposed control scheme is demonstrated using numerical simulations along with comparative study between conventional proportionalintegral-derivative (PID) control. The robustness of the proposed control scheme is also illustrated in the presence of external disturbances and parameter uncertainties.
\end{abstract}

\section{Introduction}

The underwater manipulator has turned into a critical part/tool of underwater vehicles for performing interactive tasks such as opening and closing of valves, cutting, drilling, sampling, and laying in the fields of scientific research and ocean systems engineering. Due to unstructured properties of interactive work, a good understanding of the dynamics of a robotic manipulator mounted on a moving underwater vehicle is required for autonomous manipulation tasks $[1,2]$.

The increasing in demand for more efficient, precise, and accurate underwater autonomous manipulation has induced many researches in this field, which includes the dynamic model and effective simulation of an underwater manipulator mounted on an underwater vehicle. The first attempt towards modeling of an underwater vehicle-manipulator system (UVMS) started with the development of a dynamic simulation algorithm using the Newton-Euler formulation scheme $[3,4]$. Similarly, using the Kane's method, a simplified dynamic model of UVMS was developed and verified using numerical simulations [5]. A coordinated control scheme for the UVMS using a discrete-time approximation dynamic model was developed to compensate for the tracking errors of the manipulator during vehicle motion [6]. The UVMS dynamic model was formulated using the quasi-Lagrange method $[4,7]$. A nonlinear model-based control scheme that controlled the vehicle and manipulator simultaneously was developed and investigated [8]. In addition to these studies, some of the researches were focused on estimating hydrodynamic parameters of these systems [8], the reduction of the interaction effects (dynamic coupling) between the manipulator and the vehicle [9], and the manipulability and workspace analysis of the underwater manipulator on remotely operated vehicle (ROV) [10]. Also, there have been many studies on manipulator control for enhancing efficiency of underwater manipulation, such as the force feedback control of manipulators mounted on ROVs [11], motion planning, and control of UVMS [7], computer-based control, and real-time motion compensation of UVMS [12]. The workspace-control system for the teleoperated ROV was proposed by combining the ROV master-slave systems and verified through experiments [13]. Despite of these significant researches, most intervention tasks conducted in the field still depend on operator's skills. Therefore, much demand and developments are needed in autonomous manipulation, which gives much attention to and interest in this research field. Although there have been some research attempts to overcome the parameter uncertainties on underwater robots, 
very few attempts considered the problem of uncertainties associated with time varying parameters on UVMS with manipulator motion causing dynamic coupling. In addition to the coupling effects, most of the real-time AUVs are underactuated in nature (i.e., the vehicle has fewer number of control inputs than the total number of independent states which makes the control scheme complex) which complicates the problem.

Therefore, by considering the above-mentioned issues, this paper presents the closed-form dynamic equations of an UVMS, and the interaction effects between the manipulator and the vehicle are investigated numerically based on prototype vehicle-manipulator parameters. This paper also proposes a model reference control scheme for the UVMS. This scheme enables overcoming the difficulty due to parameter uncertainties, external variations (e.g., buoyancy and payload variations), and disturbances (e.g., underwater current). In this study, the actuator and sensor characteristics such as time constant, efficiency, saturation limits, update rate, and sensor noises are considered for numerical simulations. In the vehicle dynamic modeling, off diagonal elements of the system matrices are considered as well. The effectiveness of the proposed control scheme is confirmed with specified UVMS tasks.

The remainder of this paper is organized as follows. The dynamic modeling of a general UVMS is derived in Section 2. In Section 3, a nonlinear controller for the UVMS based on the model reference control scheme is discussed and detailed performance analysis of the UVMS with different operating conditions is presented in Section 4. Finally, Section 5 holds the conclusions.

\section{Mathematical Modeling of UVMS}

2.1. Dynamic Model of the Underwater Vehicle. The dynamic model of an underwater vehicle is developed through the Newton-Euler formulation using laws of conservation of linear and angular momentum. The equations of motion of such vehicles are highly nonlinear [4] mainly due to hydrodynamic forces which act on the vehicle. Generally, the vehicle model can be written with respect to either a bodyfixed or an earth-fixed frame of reference (refer to Figure 1).

The equations of motion of an underwater vehicle having six degrees of freedom with respect to a body-fixed frame of reference can be written as [4]

$$
\mathbf{M} \dot{v}+\mathbf{C}(\nu) \nu+\mathbf{D}(\nu) \nu+\mathbf{g}(\eta)=\boldsymbol{\tau}_{V},
$$

where

$$
\begin{aligned}
& \mathbf{M}=\mathbf{M}_{\mathrm{RB}}+\mathbf{M}_{A}, \\
& \mathbf{C}(\nu)=\mathbf{C}_{\mathrm{RB}}(\nu)+\mathbf{C}_{A}(\nu), \\
& \mathbf{D}(\nu)=\mathbf{D}_{L}+\mathbf{D}_{\mathrm{Q}}|\nu| .
\end{aligned}
$$

$\mathbf{M}_{\mathrm{RB}}$ and $\mathbf{C}_{\mathrm{RB}}(\nu)$ are the rigid body mass matrix and the Coriolis and centripetal matrix, respectively. $\mathbf{M}_{A}$ and $\mathbf{C}_{A}(\nu)$ are the added mass matrix and the added Coriolis and centripetal matrix, respectively. $\mathbf{D}_{L}$ and $\mathbf{D}_{\mathrm{Q}}|\boldsymbol{\nu}|$ are the linear

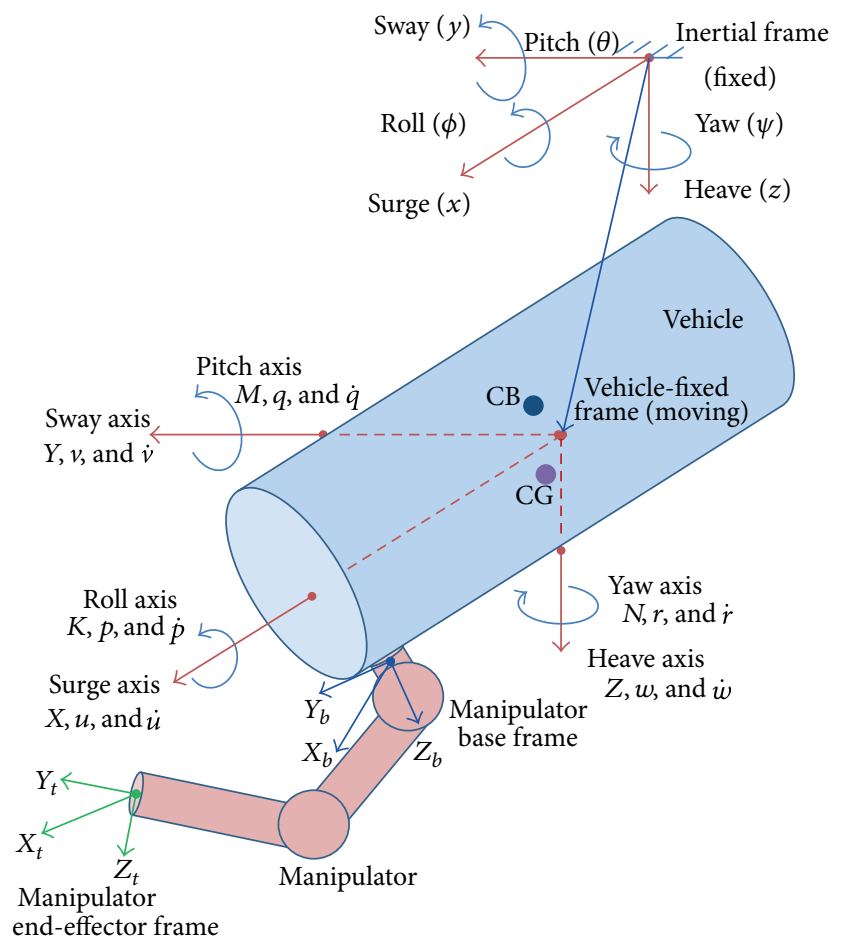

FIGURE 1: Coordinate frame arrangement of an underwater robot.

and quadratic drag matrices, respectively. $\mathbf{g}(\eta)$ is the resultant vector of gravity and buoyancy. $\boldsymbol{\tau}_{V}=\left[\begin{array}{llllll}X & Y & Z & K & M & N\end{array}\right]^{T}$ is the resultant input vector of thruster, control plane forces, and moments. $\boldsymbol{v}=\left[\begin{array}{llllll}u & v & w & p & q & r\end{array}\right]^{T}$ is the vector of linear and angular velocities in the vehicle coordinate frame. $\boldsymbol{\eta}=$ $\left[\begin{array}{llllll}x & y & z & \phi & \theta & \psi\end{array}\right]^{T}$ is the vector of absolute positions and Euler angles (roll, pitch, and yaw).

The relationship between linear and angular velocities in the vehicle frame and those in the absolute frame (refer to Figure 1) is given by

$$
\dot{\boldsymbol{\eta}}=\mathbf{J}(\eta) \boldsymbol{\nu}
$$

where $\mathbf{J}(\eta)$ is the kinematic transformation matrix and this transformation is undefined for $\theta= \pm 90^{\circ}$. However, most of the underwater vehicles are designed to operate at pitch angles well below $\pm 90^{\circ}$, and hence this limitation has no major significance here.

2.2. Kinematic Model of the Underwater Manipulator. The mathematical model of the underwater manipulator kinematics is derived in this section.

\subsubsection{Forward Kinematics of the Underwater Manipulator.} The mathematical relations of the end-effector position/tool center point (TCP) or manipulator tip position with the known joint angles are derived here. The mathematical (kinematic) descriptions of the underwater manipulator are developed based on the Denavit-Hartenberg (D-H) parameter notation [14]. The establishment of the link coordinate system as shown in Figure 2 yields the $\mathrm{D}-\mathrm{H}$ parameters 


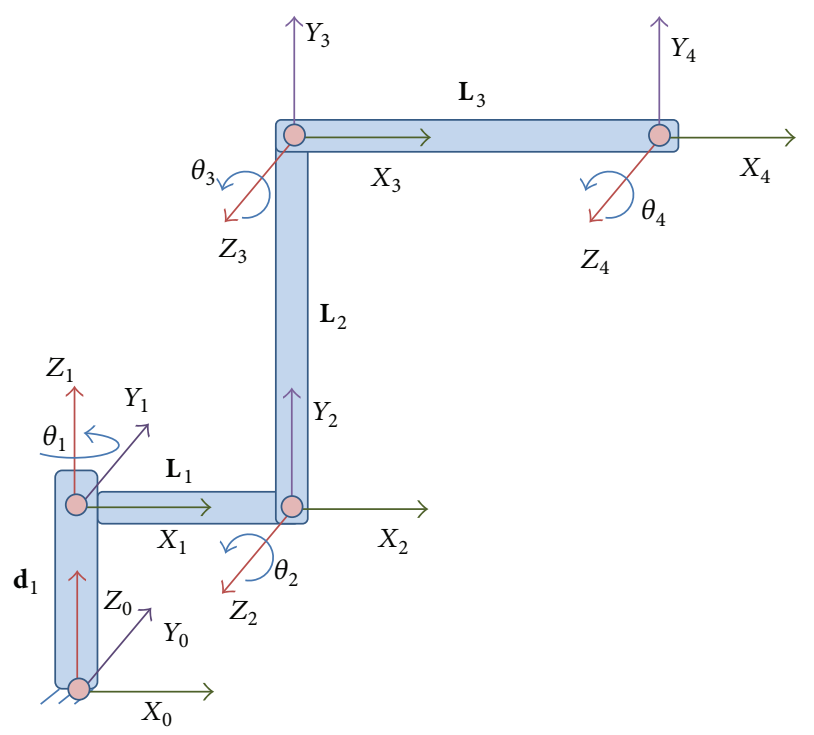

Figure 2: Establishing link coordinate systems of the manipulator.

shown in Table 1. Based on the underwater link parameters in Table 1, the homogeneous transformation matrix [14] which is derived, that specifies the location of the end effector or TCP with respect to the base coordinate system, is expressed as

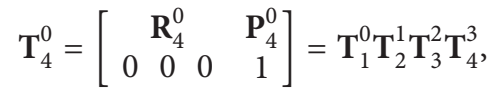

$$
\begin{aligned}
& \mathbf{T}_{k}^{k-1} \\
& =\left[\begin{array}{cccc}
\cos \theta_{k} & -\sin \theta_{k} & 0 & a_{k-1} \\
\sin \theta_{k} \cos \alpha_{k-1} & \cos \theta_{k} \cos \alpha_{k-1} & -\sin \alpha_{k-1} & -\sin \alpha_{k-1} d_{k} \\
\sin \theta_{k} \sin \alpha_{k-1} & \cos \theta_{k} \sin \alpha_{k-1} & \cos \alpha_{k-1} & \cos \alpha_{k-1} d_{k} \\
0 & 0 & 0 & 1
\end{array}\right] .
\end{aligned}
$$

The matrix $\mathbf{R}_{4}^{0}$ and the vector $\mathbf{P}_{4}^{0}=\left[\begin{array}{lll}p_{x} & p_{y} & p_{z}\end{array}\right]^{T}$ are the rotational matrix and the position vector from the base coordinates to the end effector, respectively. From the above homogeneous transformation matrix, we can obtain the end effector's position for feedback control and the elements for solving the Jacobian matrix. Based on the experimental underwater manipulator parameters (refer to Table 1), the forward kinematic solutions are obtained as follows:

$$
\begin{aligned}
& p_{x}=\cos \theta_{1}\left(L_{1}+L_{2} \cos \theta_{2}+L_{3} \cos \left(\theta_{2}+\theta_{3}\right)\right), \\
& p_{y}=\sin \theta_{1}\left(L_{1}+L_{2} \cos \theta_{2}+L_{3} \cos \left(\theta_{2}+\theta_{3}\right)\right), \\
& p_{z}=L_{2} \sin \theta_{2}+L_{3} \sin \left(\theta_{2}+\theta_{3}\right) .
\end{aligned}
$$

2.2.2. Inverse Kinematics of the Underwater Manipulator. In the workspace-control system, each joint of the underwater manipulator is controlled by the joint angle command calculated from the differential inverse kinematic solutions based
TABLE 1: D-H parameters of the 3-DOF underwater manipulator.

\begin{tabular}{lcccc}
\hline $\begin{array}{l}\text { Joint axis } \\
(k)\end{array}$ & $\begin{array}{c}\text { Link offset } \\
\left(\alpha_{k-1}\right)\end{array}$ & $\begin{array}{c}\text { Link length } \\
\left(a_{k-1}\right)\end{array}$ & $\begin{array}{c}\text { Joint distance } \\
\left(d_{k}\right)\end{array}$ & $\begin{array}{c}\text { Joint angle } \\
\left(\theta_{k}\right)\end{array}$ \\
\hline 1 & $0^{\circ}$ & 0 & 0 & $\theta_{1}$ \\
2 & $90^{\circ}$ & $L_{1}$ & 0 & $\theta_{2}$ \\
3 & $0^{\circ}$ & $L_{2}$ & 0 & $\theta_{3}$ \\
4 & $0^{\circ}$ & $L_{3}$ & 0 & $\theta_{4}=0^{\circ}$ \\
\hline
\end{tabular}

on the known Cartesian coordinates. The closed-form inverse kinematic solutions of the 3-DOF underwater manipulator are described as

$$
\begin{aligned}
& \theta_{1}=\arctan 2\left(p_{y}, p_{x}\right), \\
& \theta_{2}=\arctan 2(a b-b c, a c+b d), \\
& \theta_{3}=\arctan 2\left(\sin \theta_{3}, \cos \theta_{3}\right),
\end{aligned}
$$

where

$$
\begin{aligned}
& \cos \theta_{3}=\frac{a^{2}+b^{2}-L_{2}^{2}-L_{3}^{2}}{2 L_{2} L_{3}}, \\
& \sin \theta_{3}=\sqrt{1-\cos ^{2} \theta_{3}}, \\
& a=\frac{p_{x}}{\cos \theta_{1}}-L_{1}, \quad b=p_{z}, \\
& c=L_{3} \cos \theta_{3}+L_{2}, \quad d=L_{3} \sin \theta_{3} .
\end{aligned}
$$

2.3. Dynamic Model of the Underwater Manipulator. The dynamic model of an underwater manipulator is developed through the recursive Newton-Euler algorithm. In this work, it is assumed that the underwater manipulator links are the buildup of cylindrical elements. The effects of the hydrodynamic forces on circular cylindrical elements are described in the section, which mainly consist of added mass effects, hydrodynamic drag forces and moments, and buoyancy effects [4]. The force and moment interactions between two adjacent links are as follows:

$$
\begin{aligned}
{ }^{k} \mathbf{f}_{k}= & { }^{k} \mathbf{f}_{k+1}=\mathbf{R}_{k}^{k+1}{ }^{k+1} \mathbf{f}_{k+1}+\mathbf{F}_{k}-m_{k} \mathbf{g}_{k}+\mathbf{b}_{k}+\mathbf{p}_{k}, \\
{ }^{k} \mathbf{t}_{k}= & { }^{k} \mathbf{t}_{k+1}=\mathbf{R}_{k}^{k+1}{ }^{k+1} \mathbf{t}_{k+1}+\mathbf{d}_{k / k+1} \times \mathbf{R}_{k}^{k+1}{ }^{k+1} \mathbf{f}_{k+1} \\
& +\mathbf{d}_{k / k c} \times \mathbf{F}_{k}+\mathbf{T}_{k}+\mathbf{d}_{k / k c} \\
& \times\left(-m_{k} \mathbf{g}_{k}+\mathbf{p}_{k}\right)+\mathbf{d}_{k / k b} \times \mathbf{b}_{k}+\mathbf{n}_{k}, \\
\mathbf{p}_{k}= & \mathbf{F}_{L k}+\mathbf{F}_{D k}+\mathbf{F}_{S k}, \\
\mathbf{F}_{S k}= & \mathbf{D}_{s}^{k k} \mathbf{v}_{k}, \\
\mathbf{F}_{D k}= & \left|{ }^{k} \mathbf{v}_{k}\right|^{T} \mathbf{D}_{D}^{k}{ }^{k} \mathbf{v}_{k}, \\
\mathbf{F}_{L k}= & \left|{ }^{k} \mathbf{v}_{k}\right|^{T} \mathbf{D}_{L}^{k k} \mathbf{v}_{k}, \\
\mathbf{n}_{k}= & { }^{k} \mathbf{v}_{k} \times\left(\mathbf{M}_{k}{ }^{k} \mathbf{v}_{k}\right),
\end{aligned}
$$




$$
\begin{aligned}
& \mathbf{F}_{k}=\mathbf{M}_{k}\left({ }^{k} \mathbf{a}_{k}+{ }^{k} \boldsymbol{\alpha}_{k} \times{ }^{k} \mathbf{d}_{k / k c}+{ }^{k} \boldsymbol{\omega}_{k} \times\left({ }^{k} \boldsymbol{\omega}_{k} \times{ }^{k} \mathbf{d}_{k / k c}\right)\right), \\
& \mathbf{T}_{k}=\mathbf{I}_{k}{ }^{k} \boldsymbol{\alpha}_{k}+{ }^{k} \boldsymbol{\omega}_{k} \times\left(\mathbf{I}_{k}{ }^{k} \boldsymbol{\omega}_{k}\right), \\
& { }^{k+1} \boldsymbol{\omega}_{k+1}=\mathbf{R}_{k+1}^{k}{ }^{k} \boldsymbol{\omega}_{k}+\mathbf{z}^{T} \dot{q}_{k+1}, \\
& { }^{k+1} \boldsymbol{\alpha}_{k+1}=\mathbf{R}_{k+1}^{k}\left({ }^{k} \boldsymbol{\alpha}_{k}+{ }^{k} \boldsymbol{\omega}_{k} \times \mathbf{z}^{T} q_{k}\right)+\mathbf{z}^{T} \ddot{q}_{k+1}, \\
& { }^{k+1} \mathbf{v}_{k+1}=\mathbf{R}_{k+1}^{k}{ }^{k} \mathbf{v}_{k}+{ }^{k+1} \boldsymbol{\omega}_{k+1} \times{ }^{k+1} \mathbf{d}_{k / k c}, \\
& { }^{k+1} \mathbf{a}_{k+1}=\mathbf{R}_{k+1}^{k}{ }^{k} \mathbf{a}_{k}+{ }^{k+1} \boldsymbol{\alpha}_{k+1} \times{ }^{k+1} \mathbf{d}_{k+1 / k} \\
& +{ }^{k+1} \boldsymbol{\omega}_{k+1} \times\left({ }^{k+1} \boldsymbol{\omega}_{k+1} \times{ }^{k+1} \mathbf{d}_{k+1 / k}\right), \\
& \mathbf{M}_{k}=\left[\begin{array}{ccc}
m_{k}+\frac{\pi \rho r_{k}^{2} L_{k}}{10} & 0 & 0 \\
0 & m_{k}+\pi \rho r_{k}^{2} L_{k} & 0 \\
0 & 0 & m_{k}+\pi \rho r_{k}^{2} L_{k}
\end{array}\right] \text {, } \\
& \mathbf{I}_{k}=\left[\begin{array}{ccc}
I_{x} & 0 & 0 \\
0 & I_{y}+\frac{\pi \rho r_{k}^{2} L_{k}^{3}}{12} & 0 \\
0 & 0 & I_{z}+\frac{\pi \rho r_{k}^{2} L_{k}^{3}}{12}
\end{array}\right] \text {, }
\end{aligned}
$$

where $k$ denotes the corresponding joint axis $(k=1,2,3)$, $\mathbf{R}_{k}^{k+1}$ is the rotation matrix, $\mathbf{f}_{k}$ is the resultant force vector, $\mathbf{t}_{k}$ is the resultant moment vector, $\mathbf{p}_{k}$ is the linear and quadratic hydrodynamic friction forces, and $\mathbf{F}_{S k}, \mathbf{F}_{D k}$, and $\mathbf{F}_{L k}$ are vectors of linear skin friction, quadratic drag, and quadratic lift effects, respectively. $\mathbf{D}_{s}^{k}$ is the linear skin friction matrix, $\mathbf{D}_{L}^{k}$ is the diagonal matrix which contains lift coefficients, $\mathbf{D}_{D}^{k}$ is the diagonal matrix which contains drag coefficients, $\mathbf{g}_{k}$ is the gravity force vector, $\mathbf{b}_{k}$ is the buoyancy force vector, $\mathbf{n}_{k}$ is the hydrodynamic moment vector, $\mathbf{d}_{k / k b}$ is the vector from the center of buoyancy of the link, $\mathbf{d}_{k / k c}$ is the vector from the center of gravity of the link, $\mathbf{d}_{k / k+1}$ is the vector from joint $k$ to $k+1, \mathbf{F}_{k}$ is the vector of total forces acting at the center of mass of link, $\mathbf{T}_{k}$ is the vector of total moments acting at the center of mass of link, $\mathbf{a}_{k}$ is the linear acceleration vector, $\boldsymbol{\alpha}_{k}$ is the angular acceleration vector, $\mathbf{v}_{k}$ is the linear velocity vector, $\boldsymbol{\omega}_{k}$ is the angular velocity vector, $\mathbf{m}_{k}$ is the mass of the link, $\mathbf{M}_{k}$ is the mass and added mass matrix of the link (located at the center of mass), $\mathbf{I}_{k}$ is the moment of inertia and added moment of matrix of the link (located at the center of mass), and $\mathbf{z}^{T}$ is the unit vector along the $z$-axis.

The joint torques of each link are represented as

$$
\tau_{R k}=\mathbf{z}^{T k} \mathbf{t}_{k} \text {. }
$$

The recursive Newton-Euler dynamics algorithm for all links, symbolically, yields the equations of motion for the underwater manipulator. The result can be written as follows:

$$
\mathbf{M}_{R}(q) \ddot{\mathbf{q}}+\mathbf{C}_{R}(q, \dot{q}) \dot{\mathbf{q}}+\mathbf{D}_{R}(q, \dot{q}) \dot{\mathbf{q}}+\mathbf{g}_{R}(q)=\boldsymbol{\tau}_{R},
$$

where $\mathbf{q}$ is the vector of joint variables and $\mathbf{q}=\left[\begin{array}{lll}\theta_{1} & \theta_{2} & \theta_{3}\end{array}\right]^{T}$, $\theta_{1}, \theta_{2}, \theta_{3}$ are the joint angles of the corresponding underwater manipulator links, $\mathbf{M}_{R}(q) \ddot{\mathbf{q}}$ is the vector of inertial forces and moments of the manipulator, $\mathbf{C}_{R}(q, \dot{q}) \dot{\mathbf{q}}$ is the vector of Coriolis and centripetal effects of the manipulator, $\mathbf{D}_{R}(q, \dot{q}) \dot{\mathbf{q}}$ is the vector of damping effects of the manipulator, $\mathbf{g}_{R}(q)$ is the restoring vector of the manipulator, and $\boldsymbol{\tau}_{R}=\left[\begin{array}{lll}\tau_{R 1} & \tau_{R 2} & \tau_{R 3}\end{array}\right]^{T}$ is the vector of control inputs.

2.4. Dynamic Model of Vehicle-Manipulator System. The manipulator has an initial velocity equal to the velocity of the vehicle. This initial velocity should be accounted in the manipulator dynamics. Similarly, the weight and movement of the manipulator links will affect the vehicle dynamics (these effects are considered as disturbances) and vice versa. By considering the above issues, the vehicle and manipulator equations of motions can be written as follows:

$$
\begin{aligned}
& \mathbf{M} \dot{\boldsymbol{v}}+\mathbf{C}(\nu) \boldsymbol{v}+\mathbf{D}(\nu) \boldsymbol{v}+\mathbf{g}(\eta)=\boldsymbol{\tau}_{V}+\boldsymbol{\sigma} \\
& \quad \mathbf{M}_{R}(q) \ddot{\mathbf{q}}+\mathbf{H}_{2}^{T}(q) \dot{\boldsymbol{v}}+\mathbf{C}_{R}(q, \dot{q}) \dot{\mathbf{q}}+\mathbf{D}_{R}(q, \dot{q}) \dot{\mathbf{q}} \\
& \quad+\mathbf{g}_{R}(q)+\mathbf{C}_{V}^{T}(q, \dot{q}, v) \boldsymbol{v}+\mathbf{D}_{V}^{T}(q, \dot{q}, v) \boldsymbol{v}=\boldsymbol{\tau}_{R}
\end{aligned}
$$

where $\mathbf{H}_{2}^{T}(q) \dot{v}$ is the vector of reaction forces and moments between the vehicle and the manipulator, $\mathrm{C}_{V}^{T}(q, \dot{q}, v) v$ is the vector of Coriolis and centripetal effects due to coupling between the vehicle and the manipulator, and $\mathbf{D}_{V}^{T}(q, \dot{q}, v) v$ is the vector of quadratic drag effects due to the interaction between the vehicle and the manipulator. $\boldsymbol{\sigma}=\left[\begin{array}{ll}\boldsymbol{\sigma}_{f}^{T} & \boldsymbol{\sigma}_{m}^{T}\end{array}\right]^{T}$ is the vector which contains forces and moments disturbances due to weight and movement of the manipulator links. Consider

$$
\begin{gathered}
\boldsymbol{\sigma}_{f}=\mathbf{R}_{0}^{B 0} \mathbf{f}_{1}, \\
\boldsymbol{\sigma}_{m}=\mathbf{R}_{0}^{B ~ 0} \mathbf{t}_{1}-\mathbf{d}_{\text {vehicle/manipulator }} \times\left(\mathbf{R}_{0}^{B 0} \mathbf{f}_{1}\right),
\end{gathered}
$$

where $\mathbf{R}_{0}^{B}$ and $\mathbf{d}_{\text {vehicle/manipulator }}$ are the rotation matrix and position vector from the vehicle-fixed frame to the manipulator base frame, respectively. ${ }^{0} \mathbf{f}_{1}$ and ${ }^{0} \mathbf{t}_{1}$ are the vectors of force and moments of the base frame, respectively. These vectors are derived using (8), and these effects are function of the manipulator joint coordinates (i.e., $\ddot{\mathbf{q}}, \dot{\mathbf{q}}$, and $\mathbf{q}$ ) and the vehicle states. After evaluating the interaction forces and moments between the manipulator and the vehicle based on these vectors, the equations of motion of the vehicle can be written as

$$
\begin{aligned}
\mathbf{M} \dot{v}+ & \mathbf{H}_{1}(q) \dot{\boldsymbol{v}}+\mathbf{C}(\nu) \boldsymbol{v}+\mathbf{D}(\nu) \boldsymbol{v}+\mathbf{C}_{V}(q, \dot{q}, v) \boldsymbol{v} \\
& +\mathbf{D}_{V}(q, \dot{q}, v) \boldsymbol{v}+\mathbf{g}(\eta)+\mathbf{H}_{2}(q) \ddot{\mathbf{q}}+\mathbf{C}_{1}(q, \dot{q}) \dot{\mathbf{q}} \\
& +\mathbf{D}_{1}(q, \dot{q}) \dot{\mathbf{q}}+\mathbf{g}_{1}(q)=\boldsymbol{\tau}_{V},
\end{aligned}
$$

where $\mathbf{H}_{1}(q) \dot{\boldsymbol{v}}$ and $\mathbf{H}_{2}(q) \ddot{\mathbf{q}}$ are the added inertia effects due to the manipulator, $\mathbf{D}_{1}(q, \dot{q}) \dot{\mathbf{q}}$ is the damping effects due to the manipulator, $\mathbf{C}_{1}(q, \dot{q}) \dot{\mathbf{q}}$ is the Coriolis and centripetal effects due to the manipulator, and $\mathbf{g}_{1}(q)$ is the restoring vector due to the manipulator on the vehicle, respectively. 


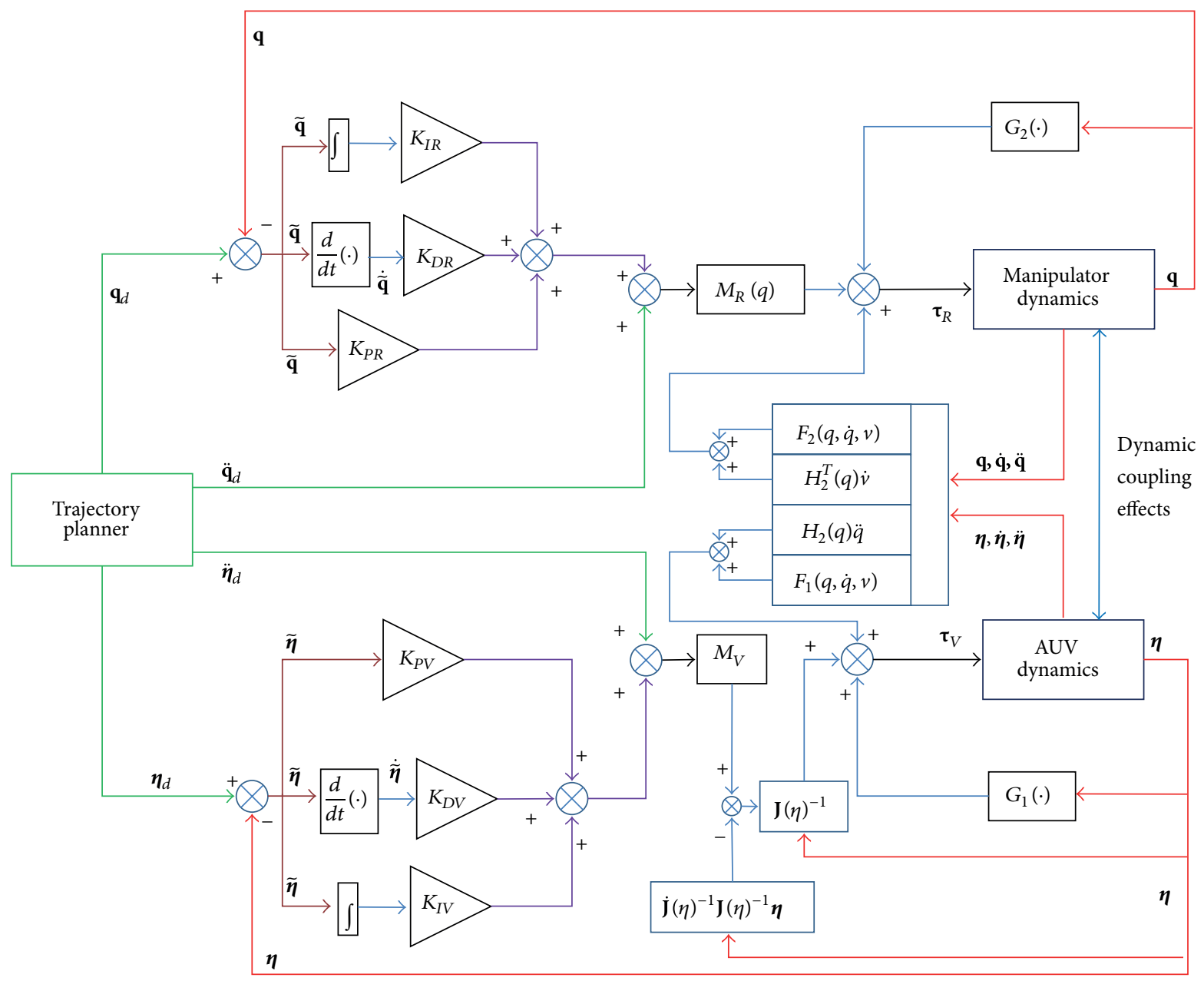

FIGURE 3: Block diagram of model reference control.

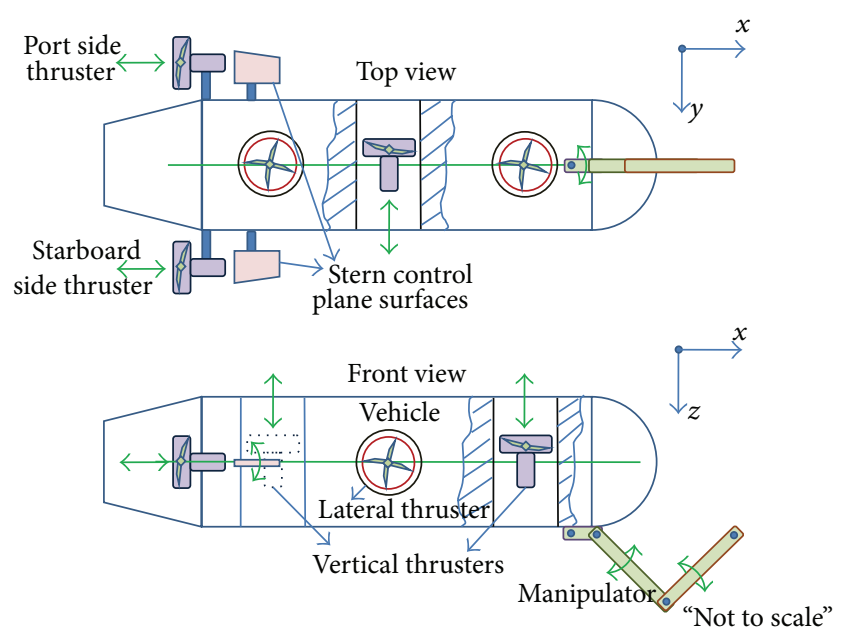

FIGURE 4: Schematic diagram of UVMS prototype model.

\section{Model Reference Control}

This section discusses the model reference control (MRC) design in terms of feedback linearization. This control scheme transforms the nonlinear system dynamics into a linear system; it compensates for all the nonlinearities in the system by introducing nonlinear elements in the input side, thus making the controller design more flexible [15-17]. Unlike traditional control, it can be thought of as "instantaneous learning" of the vehicle dynamics. The individual control laws of the underwater vehicle and the manipulator are chosen such that the tracking errors are converging to zero. The tracking errors are defined as $\widetilde{\boldsymbol{\eta}}=\boldsymbol{\eta}_{d}-\boldsymbol{\eta}$ and $\widetilde{\mathbf{q}}=\mathbf{q}_{d}-\mathbf{q}$, where $d$ denotes the desired (or reference) values. The equations of motion of the UVMS can be expressed in the form given below [15]:

$$
\begin{aligned}
& \mathbf{M}_{V} \dot{\boldsymbol{v}}+\mathbf{H}_{2}(q) \ddot{\mathbf{q}}+\mathbf{F}_{1}(q, \dot{q}, v)+\mathbf{G}_{1}(\eta, q)=\boldsymbol{\tau}_{V}, \\
& \mathbf{M}_{R}(q) \ddot{\mathbf{q}}+\mathbf{H}_{2}^{T}(q) \dot{\boldsymbol{v}}+\mathbf{F}_{2}(q, \dot{q}, v)+\mathbf{G}_{2}(q)=\boldsymbol{\tau}_{R},
\end{aligned}
$$

where $\mathbf{M}_{V}=\mathbf{M}+\mathbf{H}_{1}(q), \mathbf{F}_{1}(q, \dot{q}, v), \mathbf{F}_{2}(q, \dot{q}, v), \mathbf{G}_{2}(q)$, and $\mathbf{G}_{1}(\eta, q)$ are the vectors of the Coriolis, centripetal and damping/frictional, and restoring effects of the vehicle and the manipulator, respectively. 
The proposed control input vectors given by (18) will cancel out or compensates for the nonlinearities in the system as

$$
\begin{aligned}
& \boldsymbol{\tau}_{V}=\mathbf{M}_{V} \mathbf{u}_{V}+\mathbf{H}_{2}(q) \ddot{\mathbf{q}}+\mathbf{F}_{1}(q, \dot{q}, v)+\mathbf{G}_{1}(\eta, q), \\
& \boldsymbol{\tau}_{R}=\mathbf{M}_{R}(q) \mathbf{u}_{R}+\mathbf{H}_{2}^{T}(q) \dot{\boldsymbol{v}}+\mathbf{F}_{2}(q, \dot{q}, v)+\mathbf{G}_{2}(q),
\end{aligned}
$$

where

$$
\begin{aligned}
& \mathbf{u}_{V}=\mathbf{J}(\eta)^{-1}\left(\mathbf{u}_{\eta}-\dot{\mathbf{J}}(\eta) \mathbf{J}(\eta)^{-1} \boldsymbol{\eta}\right), \\
& \mathbf{u}_{\eta}=\ddot{\boldsymbol{\eta}}_{d}+\mathbf{K}_{p V} \widetilde{\boldsymbol{\eta}}+\mathbf{K}_{d V} \dot{\tilde{\boldsymbol{\eta}}}+\mathbf{K}_{i V} \int \widetilde{\boldsymbol{\eta}} d t, \\
& \mathbf{u}_{R}=\ddot{\mathbf{q}}_{d}+\mathbf{K}_{p R} \widetilde{\mathbf{q}}+\mathbf{K}_{d R} \dot{\tilde{\mathbf{q}}}+\mathbf{K}_{i R} \int \tilde{\mathbf{q}} d t .
\end{aligned}
$$

$\mathbf{K}_{p V}, \mathbf{K}_{i V}$, and $\mathbf{K}_{d V}$ are the proportional, integral, and derivative gain matrices of the vehicle controller, respectively, and $\mathbf{K}_{p R}, \mathbf{K}_{i R}$, and $\mathbf{K}_{d R}$ are the proportional, integral, and derivative gain matrices of the manipulator controller, respectively. Block diagram representation of the proposed UVMS controller is presented in Figure 3.

The above-mentioned control vectors lead to exponential stable error dynamics of the vehicle and manipulator which are given as

$$
\begin{aligned}
& \ddot{\widetilde{\boldsymbol{\eta}}}+\mathbf{K}_{p V} \widetilde{\boldsymbol{\eta}}+\mathbf{K}_{d V} \dot{\tilde{\boldsymbol{\eta}}}+\mathbf{K}_{i V} \int \tilde{\boldsymbol{\eta}} d t=0, \\
& \ddot{\widetilde{\mathbf{q}}}+\mathbf{K}_{p R} \widetilde{\mathbf{q}}+\mathbf{K}_{d R} \dot{\widetilde{\mathbf{q}}}+\mathbf{K}_{i R} \int \widetilde{\mathbf{q}} d t=0,
\end{aligned}
$$

which implies that tracking errors are converging to zero (i.e., $\widetilde{\boldsymbol{\eta}} \rightarrow 0$ and $\widetilde{\mathbf{q}} \rightarrow 0$ ) and the UVMS follows the given desired trajectory [18].

However, the above-proposed control scheme assumed that there are no disturbances and no uncertainties associated with the system. If the system uncertainties and external disturbances are considered for the analysis, the system error dynamics become

$$
\begin{aligned}
& \ddot{\widetilde{\boldsymbol{\eta}}}+\mathbf{K}_{p V} \widetilde{\boldsymbol{\eta}}+\mathbf{K}_{d V} \dot{\tilde{\boldsymbol{\eta}}}+\mathbf{K}_{i V} \int \tilde{\boldsymbol{\eta}} d t=\mathbf{M}_{V}{ }^{-1} \boldsymbol{\tau}_{d V}, \\
& \ddot{\tilde{\mathbf{q}}}+\mathbf{K}_{p R} \widetilde{\mathbf{q}}+\mathbf{K}_{d R} \dot{\widetilde{\mathbf{q}}}+\mathbf{K}_{i R} \int \widetilde{\mathbf{q}} d t=\mathbf{M}_{R}{ }^{-1} \boldsymbol{\tau}_{d R},
\end{aligned}
$$

where $\boldsymbol{\tau}_{d V}$ and $\boldsymbol{\tau}_{d R}$ are the disturbance vector (which combines the internal disturbances such as effect of parameter uncertainties and external disturbances, namely, underwater current, etc.) of the vehicle and manipulator controller, respectively.

The closed-loop characteristic equation or polynomial is given as

$$
\begin{aligned}
& \Delta_{c V}(s)=\left|s^{2} \mathbf{I}+s \mathbf{K}_{d V}+\mathbf{K}_{p V}+\frac{\mathbf{K}_{i V}}{s}\right| \\
& \Delta_{c R}(s)=\left|s^{2} \mathbf{I}+s \mathbf{K}_{d R}+\mathbf{K}_{p R}+\frac{\mathbf{K}_{i R}}{s}\right| .
\end{aligned}
$$

The control gain matrices are chosen as diagonal and positive definite matrices, such as

$$
\begin{array}{ll}
\mathbf{K}_{d V}=\operatorname{diag}\left(k_{d V i}\right), & \mathbf{K}_{p V}=\operatorname{diag}\left(k_{p V i}\right), \\
\mathbf{K}_{i V}=\operatorname{diag}\left(k_{i V i}\right), & \mathbf{K}_{d R}=\operatorname{diag}\left(k_{d R i}\right), \\
\mathbf{K}_{p R}=\operatorname{diag}\left(k_{p R i}\right), & \mathbf{K}_{i R}=\operatorname{diag}\left(k_{i R i}\right) .
\end{array}
$$

Therefore, the closed-loop characteristic polynomial becomes

$$
\begin{aligned}
& \Delta_{c V}(s)=\prod_{i=1}^{6}\left(s^{2}+s k_{d V i}+k_{p V i}+\frac{k_{i V i}}{s}\right), \\
& \Delta_{c R}(s)=\prod_{i=1}^{n}\left(s^{2}+s k_{d R i}+k_{p R i}+\frac{k_{i R i}}{s}\right),
\end{aligned}
$$

and the system error is asymptotically stable as long as the controller gain values are all positive. Therefore, as long as the disturbance vectors are bounded, the system tracking errors are converged to zero. In other words, the system follows the given desired trajectory. Moreover, the controller gain matrices have been chosen in such a way that

$$
\begin{array}{ll}
\lambda_{\min }\left\{\mathbf{K}_{p V}\right\}>\left\|\frac{\partial \boldsymbol{\tau}_{d V}}{\partial \widetilde{\boldsymbol{\eta}}}\right\|, & \lambda_{\min }\left\{\mathbf{K}_{d V}\right\}>\left\|\frac{\partial \boldsymbol{\tau}_{d V}}{\partial \dot{\tilde{\boldsymbol{\eta}}}}\right\|, \\
\lambda_{\min }\left\{\mathbf{K}_{p R}\right\}>\left\|\frac{\partial \boldsymbol{\tau}_{d R}}{\partial \widetilde{\mathbf{q}}}\right\|, & \lambda_{\min }\left\{\mathbf{K}_{d R}\right\}>\left\|\frac{\partial \boldsymbol{\tau}_{d R}}{\partial \dot{\tilde{\mathbf{q}}}}\right\| .
\end{array}
$$

Hence, $\widetilde{\boldsymbol{\eta}}=0$ and $\widetilde{\mathbf{q}}=0$ for all $t \geq 0$ are its unique solution, and it can be observed that the system tracking errors are converging to zero asymptotically. The proposed controller analysis does not contain explicit treatment of the parametric uncertainties and external disturbance while taking temporal derivative along the closed-loop system trajectories, and it is left for further research and analysis.

\section{Performance Analysis of the UVMS}

4.1. Description of the Simulated System. We have accomplished widespread computer-based numerical simulations to explore the total performance of the proposed UVMS and the model reference (direct adaptive) control scheme. The UVMS used for this study consists of six degrees of freedom (DOF) vehicle and a 3-DOF spatial manipulator; the UVMS arrangement in conceptual design is presented in Figure 4. The vehicle is a cylindrical shape (torpedo fish shaped) cruising type with the length of $1.1 \mathrm{~m}$ and the diameter of $0.25 \mathrm{~m}$. The mass of the vehicle is $39 \mathrm{~kg}$. The vehicle has five thrusters in total, two propulsion thrusters (in the stern side), two vertical thrusters, and one lateral thruster. Therefore, surge, sway, and heave motions are controlled through these thrusters actuation directly, and, however, yaw and pitch motions are controlled by differential actuation of propulsion and vertical thrusters. Although roll motion is unactuated, it can be controlled itself due to restoring effects [19-21]. It is known that a necessary condition for a submersible body to 
be stable is that the centre of gravity (CG) should lie below the centre of buoyancy (CB). For this, the vehicle is trimmed (using a float) to have the CB almost vertically above the CG and made to be nearly neutral buoyant. Here the roll motion has nonzero components of restoring moments that represent self-restoring torque for the roll direction. With nonzero restoring terms, it may be possible to find continuous state feedback laws to stabilize the system as a whole. In addition to this, without loss of generality, we can assume that the damping terms of nonactuated states are sufficiently larger than their inertia terms which means that the hydrodynamic restoring forces and torques are large enough to stabilize the nonactuated (roll) states (zero dynamics), which is a common property for AUVs [19]. This means that the underwater robot can be exponentially stabilized by the actuated state controls alone.

The manipulator links are cylindrical in shape, and the radii of links 1,2 , and 3 are $0.1 \mathrm{~m}, 0.05 \mathrm{~m}$, and $0.05 \mathrm{~m}$, respectively. The lengths of links 1,2 , and 3 are $0.1 \mathrm{~m}, 0.2 \mathrm{~m}$, and $0.2 \mathrm{~m}$, respectively. The link masses (along with oil conserved motors) are $0.39 \mathrm{~kg}, 1.57 \mathrm{~kg}$, and $1.57 \mathrm{~kg}$, respectively. Here, the links are considered as cylindrical shape because such shape provides uniform hydrodynamic reactions and is one of the primary candidates for possible link geometry for underwater manipulators. Although it is cylindrical, our mathematical framework does not depend on any particular shapes; it can be easily accommodated by any other shapes. Indeed, cylindrical underwater manipulators are available in the present market [22]. Hydrodynamic parameters of the UVMS are estimated using empirical relations based on strip theory. This method is verified using the available literature; therefore, these values are reliable and can be used for further developments. Some parameters like inertia, centre of gravity, and centre of buoyancy are calculated from the geometrical design of the UVMS. The total parameter list of the UVMS is given in Tables 2 and 3.

We have compared our results with those of traditional PID controller; the following control vectors were considered for the vehicle and the manipulator and are given by

$$
\begin{aligned}
& \boldsymbol{\tau}_{V}=\mathbf{K}_{D V} \dot{\tilde{\boldsymbol{\eta}}}+\mathbf{K}_{P V} \widetilde{\boldsymbol{\eta}}+\mathbf{K}_{I V} \int \widetilde{\boldsymbol{\eta}} d t, \\
& \boldsymbol{\tau}_{R}=\mathbf{K}_{D R} \dot{\tilde{\mathbf{q}}}+\mathbf{K}_{P R} \widetilde{\mathbf{q}}+\mathbf{K}_{I R} \int \widetilde{\mathbf{q}} d t,
\end{aligned}
$$

where $\mathbf{K}_{P V}, \mathbf{K}_{I V}, \mathbf{K}_{D V}, \mathbf{K}_{P R}, \mathbf{K}_{I R}$, and $\mathbf{K}_{D R}$ are the proportional, integral, and derivative gain matrices of the vehicle and the manipulator, respectively.

4.2. Description of the Tasks. Three different tasks with four different cases have been considered for the simulations. The first task is that the vehicle is commanded to track a given $3 \mathrm{D}$ trajectory without and with the manipulator (at rest or its home position), and in this condition the vehicle accelerates for the initial $20 \mathrm{~s}$ and attains the vehicle forward speed of $1 \mathrm{~m} / \mathrm{s}(\approx 2$ knots) and for the final $20 \mathrm{~s}$ the vehicle decelerates to zero speed. The particular desired 3D trajectory (refer to Figure 5) has been chosen for this performance analysis, because this trajectory consists of all four main motions such
TABLE 2: The parameter list of the UVMS.

\begin{tabular}{lc}
\hline Parameters & Values \\
\hline Mass of the vehicle $\left(m_{v}\right)$ & $39 \mathrm{~kg}$ \\
Length of the vehicle $\left(L_{v}\right)$ & $1.1 \mathrm{~m}$ \\
Diameter of the vehicle $\left(d_{v}\right)$ & $0.25 \mathrm{~m}$ \\
Moment of inertia of the vehicle & \\
$I_{x}$ & $0.34 \mathrm{kgm}^{2}$ \\
$I_{y}$ & $3.71 \mathrm{kgm}^{2}$ \\
$I_{z}$ & $3.71 \mathrm{kgm}^{2}$ \\
Centre of gravity $(\mathrm{CG})=\left(x_{G}, y_{G}, z_{G}\right)$ & $(0,0,0) \mathrm{m}$ \\
Centre of buoyancy $(\mathrm{CB})=\left(x_{B}, y_{B}, z_{B}\right)$ & $(0,0,0.015) \mathrm{m}$ \\
Mass of the underwater manipulator links & \\
$m_{1}$ & $0.3927 \mathrm{~kg}$ \\
$m_{2}$ & $1.5708 \mathrm{~kg}$ \\
$m_{3}$ & $1.5708 \mathrm{~kg}$ \\
Underwater manipulator link lengths & \\
$L_{1}$ & $0.05 \mathrm{~m}$ \\
$L_{2}$ & $0.20 \mathrm{~m}$ \\
$L_{3}$ & $0.20 \mathrm{~m}$ \\
Underwater manipulator link radii & \\
$r_{1}$ & $0.10 \mathrm{~m}$ \\
$r_{2}$ & $0.05 \mathrm{~m}$ \\
$r_{3}$ & $0.05 \mathrm{~m}$ \\
Coefficient of drag $\left(C_{D}\right)$ & 1.25 \\
\hline
\end{tabular}

TABLE 3: The values of hydrodynamic coefficients calculated using empirical relations based on strip theory.

\begin{tabular}{lccc}
\hline \multicolumn{4}{c}{ Vehicle added mass derivatives } \\
\hline$X_{\dot{u}}$ & $-1.17 \mathrm{~kg}$ & $K_{\dot{p}}$ & $-0.1 \mathrm{kgm} / \mathrm{rad}$ \\
$Y_{\dot{v}}$ & $-34.84 \mathrm{~kg}$ & $M_{\dot{w}}$ & $-1.03 \mathrm{kgm}$ \\
$Y_{\dot{r}}$ & $1.04 \mathrm{kgm}$ & $M_{\dot{q}}$ & $-2.66 \mathrm{kgm} / \mathrm{rad}$ \\
$Z_{\dot{w}}$ & $-34.83 \mathrm{~kg}$ & $N_{\dot{v}}$ & $-1.04 \mathrm{kgm}$ \\
$Z_{\dot{q}}$ & $-1.04 \mathrm{kgm}$ & $N_{\dot{r}}$ & $-2.65 \mathrm{kgm} / \mathrm{rad}$ \\
\hline \multicolumn{4}{c}{} \\
\hline$X_{u|u|}$ & Vehicle hydrodynamic parameters \\
$X_{v|v|}$ & $-7.365 \mathrm{~kg} / \mathrm{m}$ & $X_{u}$ & $-2.12 \mathrm{~kg} / \mathrm{s}$ \\
$X_{w|w|}$ & $-0.737 \mathrm{~kg} / \mathrm{m}$ & $X_{v}$ & $-0.31 \mathrm{~kg} / \mathrm{s}$ \\
$X_{q|q|}$ & $-0.737 \mathrm{~kg} / \mathrm{m}$ & $X_{w}$ & $-0.31 \mathrm{~kg} / \mathrm{s}$ \\
$X_{r|r|}$ & $-1.065 \mathrm{kgm} / \mathrm{rad}$ & $X_{q}$ & $-0.51 \mathrm{kgm} / \mathrm{s}$ \\
$Y_{v|v|}$ & $-1.065 \mathrm{kgm} / \mathrm{rad}$ & $X_{r}$ & $-0.51 \mathrm{kgm} / \mathrm{s}$ \\
$Y_{r|r|}$ & $-112.2 \mathrm{~kg} / \mathrm{m}$ & $Y_{v}$ & $-62.45 \mathrm{~kg} / \mathrm{s}$ \\
$Z_{w|w|}$ & $0.250 \mathrm{kgm} / \mathrm{rad}$ & $Y_{r}$ & $0.12 \mathrm{kgm} / \mathrm{s}$ \\
$Z_{q|q|}$ & $-112.2 \mathrm{~kg} / \mathrm{m}$ & $Z_{w}$ & $-62.45 \mathrm{~kg} / \mathrm{s}$ \\
$K_{p|p|}$ & $-0.250 \mathrm{kgm} / \mathrm{rad}$ & $Z_{q}$ & $0.12 \mathrm{kgm} / \mathrm{s}$ \\
$M_{w|w|}$ & $-0.5975 \mathrm{kgm} / \mathrm{rad}$ & $K_{p}$ & $-0.3125 \mathrm{kgm} / \mathrm{s}$ \\
$M_{q|q|}$ & $2.244 \mathrm{kgm} / \mathrm{rad}$ & $M_{w}$ & $1.2 \mathrm{kgm} / \mathrm{s}$ \\
$N_{v|v|}$ & $-119.5 \mathrm{kgm} / \mathrm{rad}$ & $M_{q}$ & $-59.75 \mathrm{kgm} / \mathrm{s}$ \\
\hline & $-2.244 \mathrm{kgm} / \mathrm{rad}$ & $N_{v}$ & $1.2 \mathrm{kgm} / \mathrm{s}$ \\
\hline & & &
\end{tabular}

as sway in and out and heave up and down. The average value of sway velocity is $0.25 \mathrm{~m} / \mathrm{s}(\approx 0.5 \mathrm{knots})$ (same value for heave as well). This task is performed to identify the influences of 


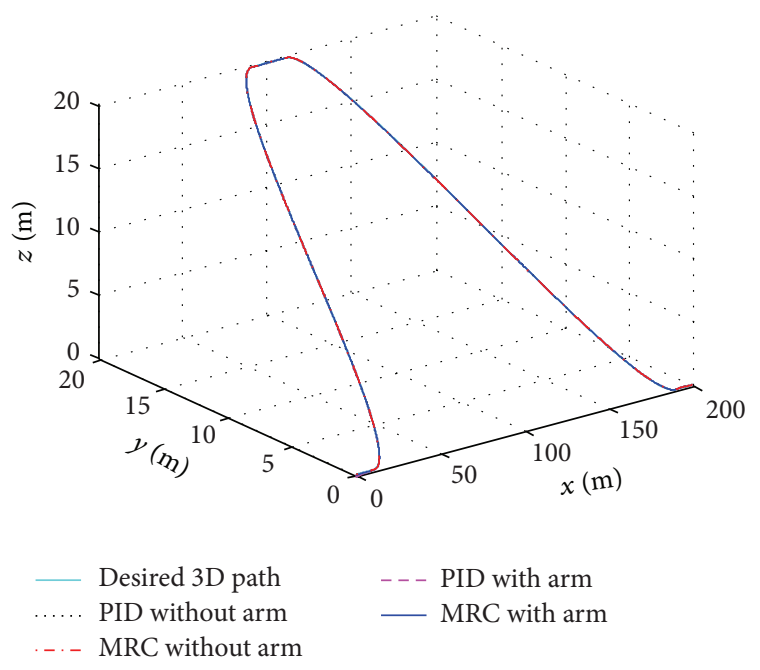

FIGURE 5: Comparative trajectory tracking control results.

the underwater manipulator effects during vehicle tracking control. In the second task, the vehicle is free to move (no control action involved) and the manipulator has a certain repeated desired trajectory. This task is mainly performed to identify the vehicle behavior during manipulator motion. In all the above tasks, the initial velocities and positions of the vehicle and the manipulator are chosen to be zero, and the initial desired and actual positions and orientations are the same. In the third task, two basic trajectories for the manipulator have been chosen for simulations, a circular trajectory (in 3D space) and a rectangular trajectory (in $y z$ plane) along with a straight line in $3 \mathrm{D}$ space. This is for the reason that most of the underwater intervention tasks involve these types of trajectories and also we can arrange any spatial trajectory by combining these trajectories. For the rectangular trajectory, we have selected a cubic polynomial function as the trajectory function, which imposes that the trajectory is continuous and smooth.

In the performance analysis, the sensory noises in position and orientation measurements are introduced and we have considered Gaussian noise of $0.01 \mathrm{~m}$ mean and $0.01 \mathrm{~m}$ standard deviation for the position measurements and $0.2^{\circ}$ mean and $0.2^{\circ}$ standard deviation for the orientation measurements. The thruster dynamic characteristics are also incorporated in the simulations. All the vehicle thrusters are considered as an identical one, and the following thruster characteristics are considered for the analysis: the thruster response delay time is $200 \mathrm{~ms}$, efficiency is $95 \%$ and saturation limits are $\pm 20 \mathrm{~N}$. The vehicle surge thrusters are located at $0.5 \mathrm{~m}$ from the body centre along the $x$-axis and offset each side $0.3 \mathrm{~m}$ from $y$-axis. The lateral thruster is exactly located on the body centre, and vertical thrusters are placed on the $x$ axis $0.4 \mathrm{~m}$ apart from the centre at each side. The controller update rate and sensor response time are considered as $100 \mathrm{~ms}$ each.

4.3. Results and Discussions. Here, numerical simulation results are shown and discussed to investigate the dynamic
TABLE 4: Controller parameter settings for simulations.

\begin{tabular}{lc}
\hline Vehicle controller parameters & Values \\
\hline$K_{p V}$ & $\operatorname{diag}(4.2,2.2,2.5,0,25,27)$ \\
$K_{d V}$ & $\operatorname{diag}(2.25,1.2,1.3,0,15,13)$ \\
$K_{i V}$ & $\operatorname{diag}(0.5,0.2,0.3,0,2.5,2.2)$ \\
$K_{P V}$ & $\operatorname{diag}(180,130,150,0,150,130)$ \\
$K_{D V}$ & $\operatorname{diag}(80,100,100,0,80,90)$ \\
$K_{I V}$ & $\operatorname{diag}(15,12,15,0,16,18)$ \\
\hline Manipulator controller parameters & $\operatorname{Values}$ \\
\hline$K_{p R}$ & $\operatorname{diag}(5,10,12)$ \\
$K_{d R}$ & $\operatorname{diag}(3,5,8)$ \\
$K_{i R}$ & $\operatorname{diag}(0.1,0.2,0.2)$ \\
$K_{P R}$ & $\operatorname{diag}(60,80,75)$ \\
$K_{D R}$ & $\operatorname{diag}(35,40,50)$ \\
$K_{I R}$ & $\operatorname{diag}(1,1.5,2)$ \\
\hline
\end{tabular}

coupling effects and the effectiveness of the proposed modelbased control scheme, which is expected to provide an intuitive, promising prospective of the proposed approach.

\subsubsection{Underwater Vehicle Tracking a Given 3D Trajectory} with the Underwater Manipulator. In this case, as mentioned earlier, the vehicle is commanded to follow a given $3 \mathrm{D}$ desired trajectory. The desired 3D trajectory is given in Figure 5. The controller gain values are tuned based on the genetic algorithms (GA) for minimizing the integral squared error (ISE) as the cost/objective function [23]. Here, we have considered both the model reference control and the PID control performance to be almost equal in the ideal situation, which makes the controller performance comparison quite reasonable and gives much better way of understanding. Based on the GA tuning method, the controller parameters are obtained and given in Table 4. The same set of controller parameters are used throughout the entire performance analysis.

Initially, the performance analysis is carried out for the vehicle trajectory tracking without manipulator inclusion. The actual simulated trajectories based on MRC and PID are presented in Figure 5. These results show that both controllers are good enough to track the given trajectory, and the tracking errors are within the design limits (error limits for the vehicle during trajectory tracking are $\pm 0.2 \mathrm{~m}$ and $\pm 0.2^{\circ}$ in positions and orientations, resp.) as well. The tracking errors are presented in Figures 6, 7, 8, 9, 10, and 11. In the second set of simulations, the manipulator is included and the inclusion effects during trajectory tracking of the vehicle are considered. Here, the manipulator links are folded and arranged in such a way that the influences on the vehicle dynamics are small (i.e., manipulator joint angles are $\theta_{1}=$ $180^{\circ}, \theta_{2}=0^{\circ}$, and $\left.\theta_{3}=180^{\circ}\right)$. The manipulator inclusion shows that there are significant performance changes in the vehicle trajectory tracking, especially during the heave and sway motion tracking. The heave, roll, and pitch motions of the vehicle are greatly influenced by the underwater manipulator inclusion, which are replicated in the results 


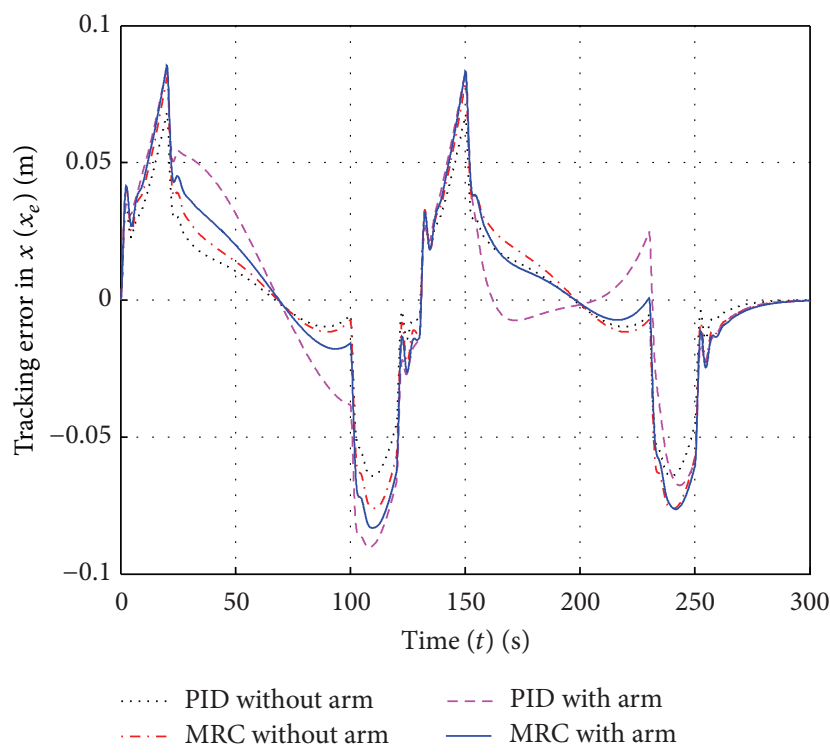

FIGURE 6: Tracking errors in $x$ position for a given 3D trajectory.

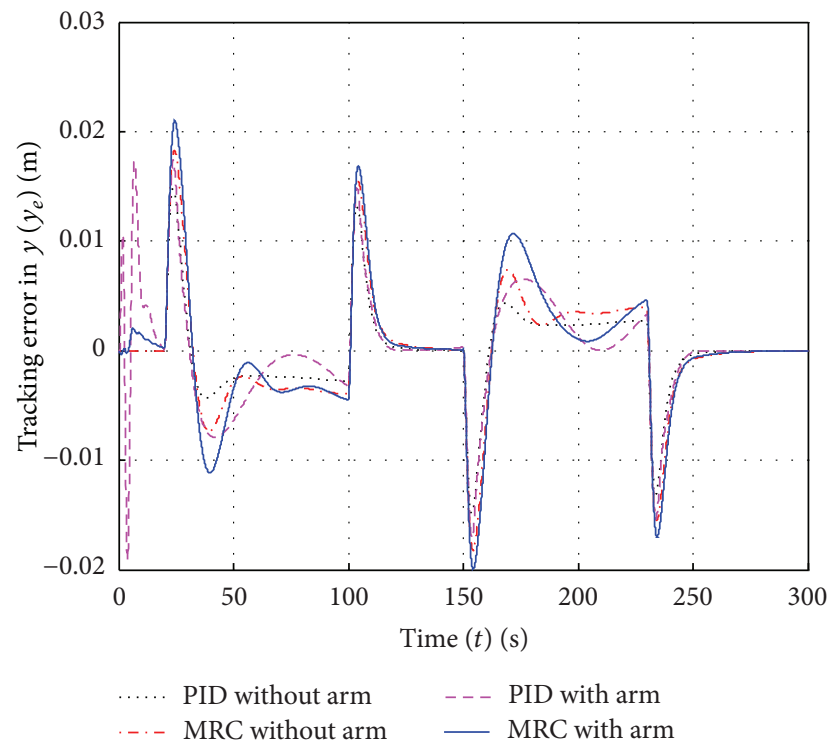

FIGURE 7: Tracking errors in $y$ position for a given 3D trajectory.

(refer to Figures 6 to 11). However, the manipulator inclusion effects are better adapted in the model reference control than in the PID control. As can be observed from Figures 8 and 10 , the manipulator inclusion causes an initial pitch angle variation drifts the vehicle in the heave direction during the straight line motion (during acceleration stage). However, these unwanted effects are compensated successfully in the model reference control and therefore tracking errors do not exceed their design limits.

4.3.2. Manipulator following a Given Desired 3D Trajectory with the Freely Moving Underwater Vehicle (without Vehicle Control). As mentioned earlier in this section, this task is mainly to address the interaction effects and their influences

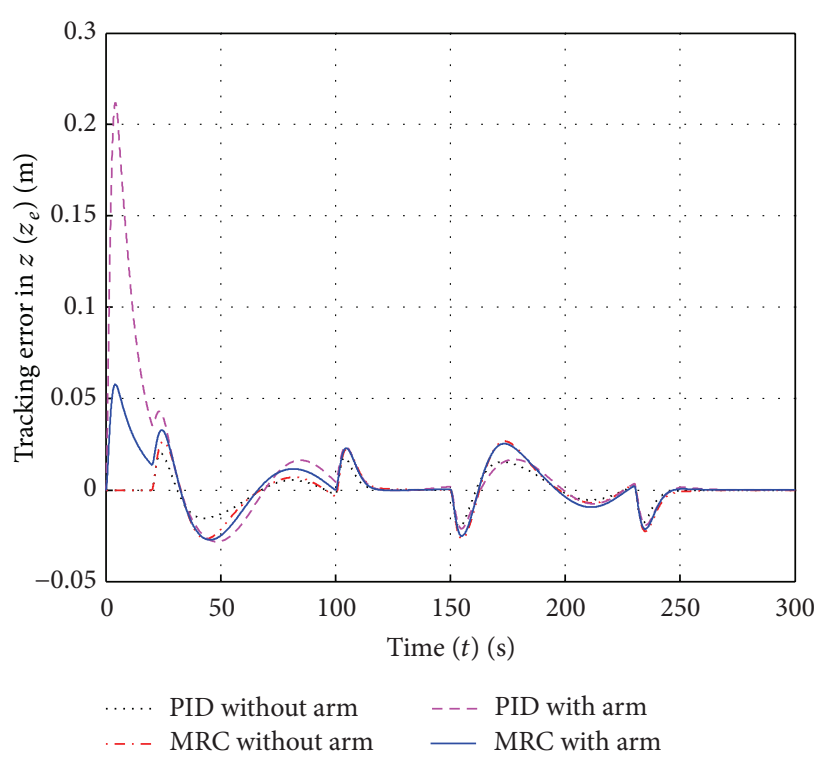

FIGURE 8: Tracking errors in $z$ position for a given 3D trajectory.

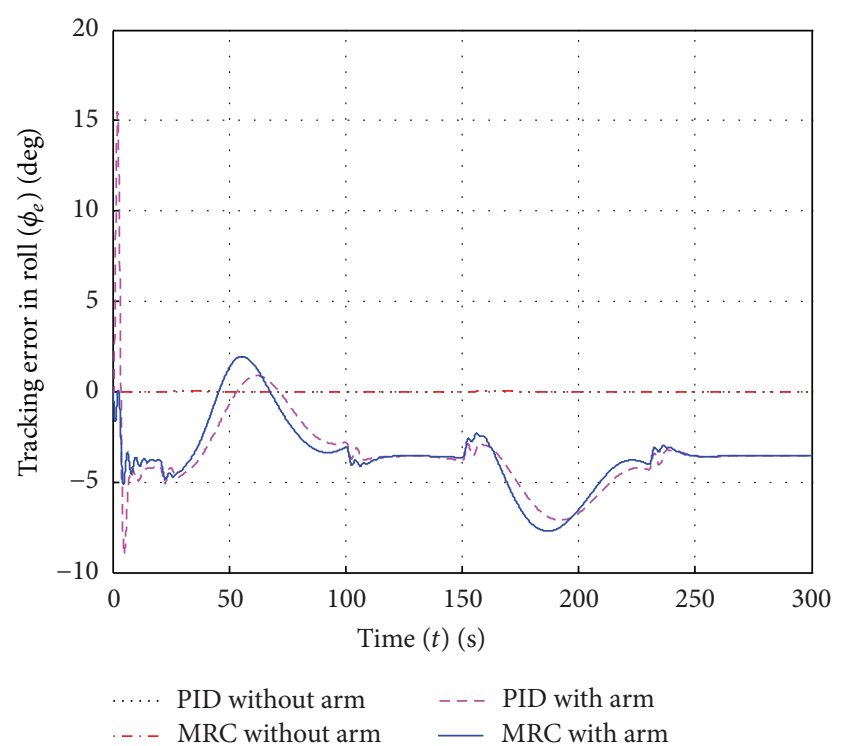

FIGURE 9: Tracking errors in roll for a given 3D trajectory.

on the UVMS. For this task, the vehicle controller is removed, and only the underwater manipulator follows a given desired continuous trajectory. The desired values of joint positions are as follows: $\theta_{1 d}=90^{\circ} \sin (0.1 t), \theta_{2 d}=90^{\circ}$, and $\theta_{3 d}=$ $-45^{\circ}$. The simulation results of this condition are presented in Figures 12, 13, 14, 15, and 16. From the results, it is observed that the vehicle is started, getting drifted from its initial position, and it is moving forward with an average velocity of $0.02 \mathrm{~m} / \mathrm{s}$ (approximately) for the repeated underwater manipulator movements. It shows that the manipulator links are affected due to the dynamic coupling effects. From the results, it is observed that the vehicle swims during manipulator movements if the vehicle does not have any control action. These errors and forward speed are increasing 


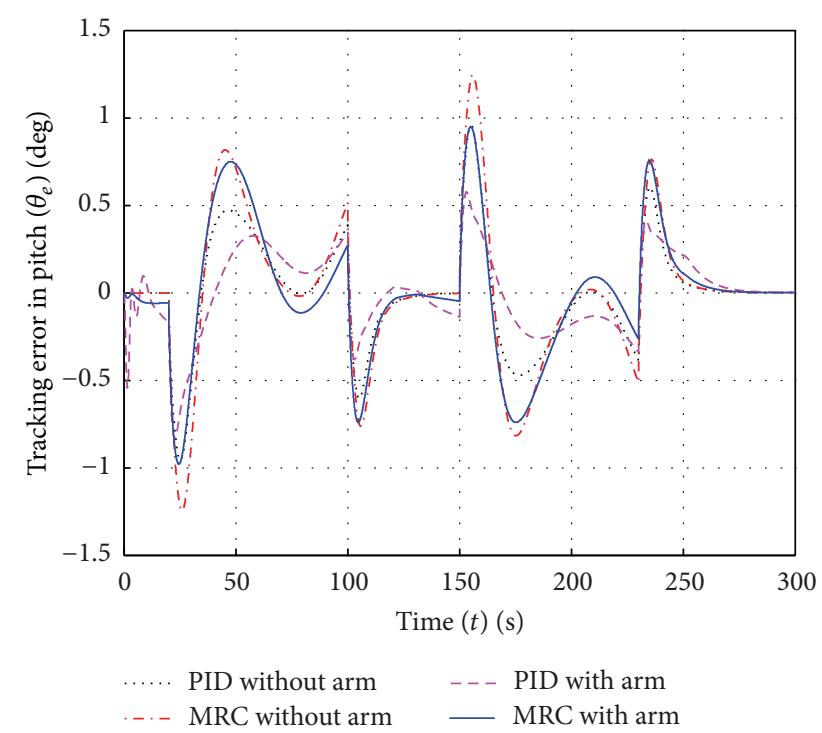

FIGURE 10: Tracking errors in pitch for a given 3D trajectory.

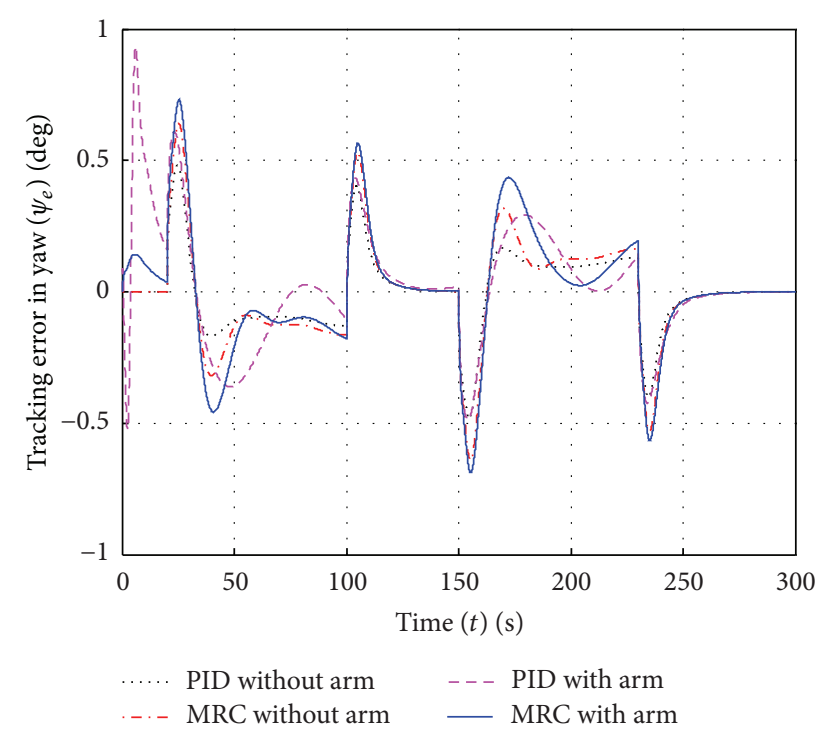

FIGURE 11: Tracking errors in yaw for a given 3D trajectory.

with the manipulator oscillation frequency and their joint angle magnitudes. In addition, the direction of the vehicle's movement varies depending on the manipulator positions and its direction of movements. These results can be extended to the work towards swimming robots and their dynamic modeling aspects.

4.3.3. Underwater Manipulator following a Given Desired Circular Trajectory with Vehicle Control. The circular trajectory chosen for the analysis has $0.2 \mathrm{~m}$ in diameter and an average speed of $0.0125 \mathrm{~m} / \mathrm{s}$. These parameters are chosen with the consideration of manipulator actuators, workspace, and control. The results from computer simulations are presented in Figures 15-18. In Figure 15, both desired and actual task space trajectories are plotted, and the task space tracking errors are given in Figures 16-18. From the results,

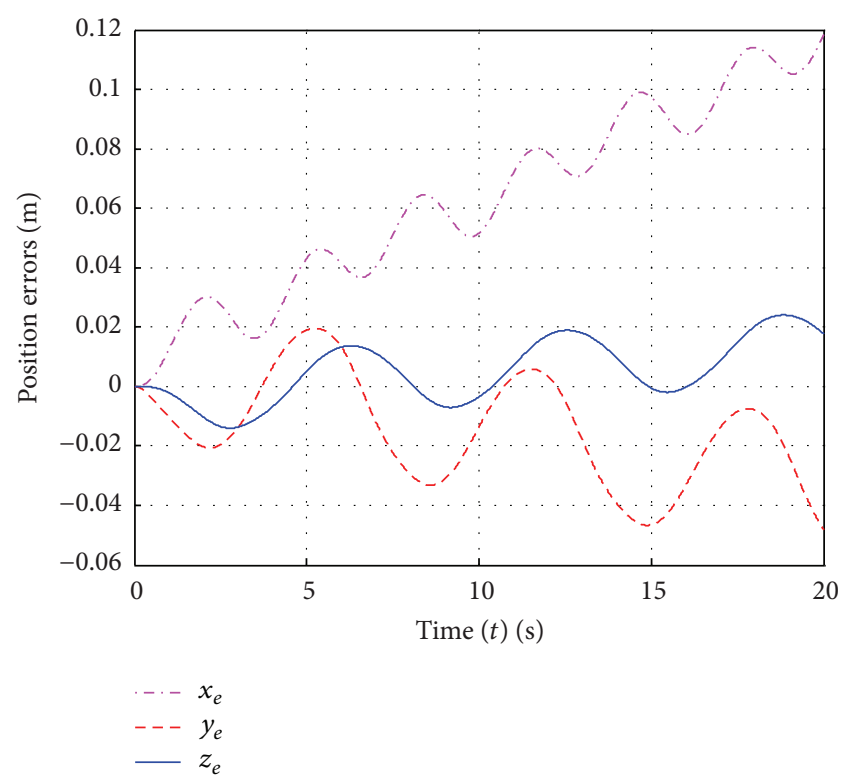

FIGURE 12: Tracking errors in the vehicle positions (when manipulator has motion).

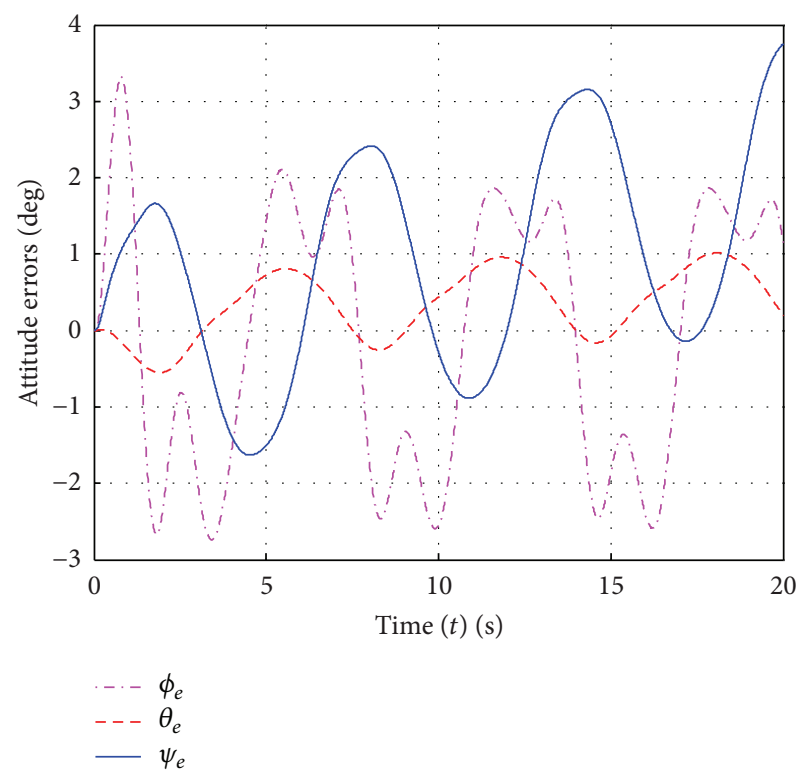

FIGURE 13: Tracking errors in the vehicle orientations (when the vehicle has no control and the manipulator has repeated motion).

it is observed that the manipulator tip tracks the given desired task space trajectories quite satisfactorily, in both PID and MRC schemes in the ideal conditions (no disturbances, no uncertainties, etc.). Then, in order to demonstrate the adaptability and robustness of the proposed controller, an uncertain condition is considered for the simulations. The following conditions are considered for the uncertain condition analysis:

(i) the UVMS parameters are assumed to be inaccurate (about 10\% for each parameters);

(ii) the manipulator carries a payload of $1 \mathrm{~kg}$ at its tip; 


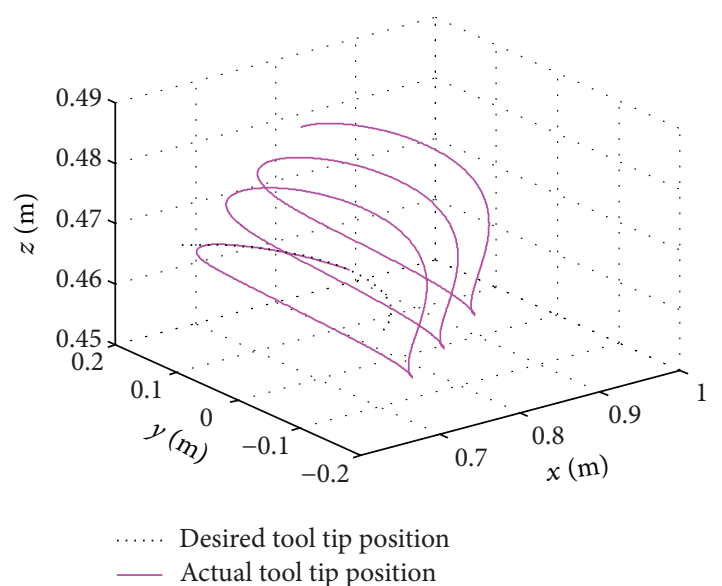

FIGURE 14: Manipulator tip position in 3D space (when the vehicle has no control).

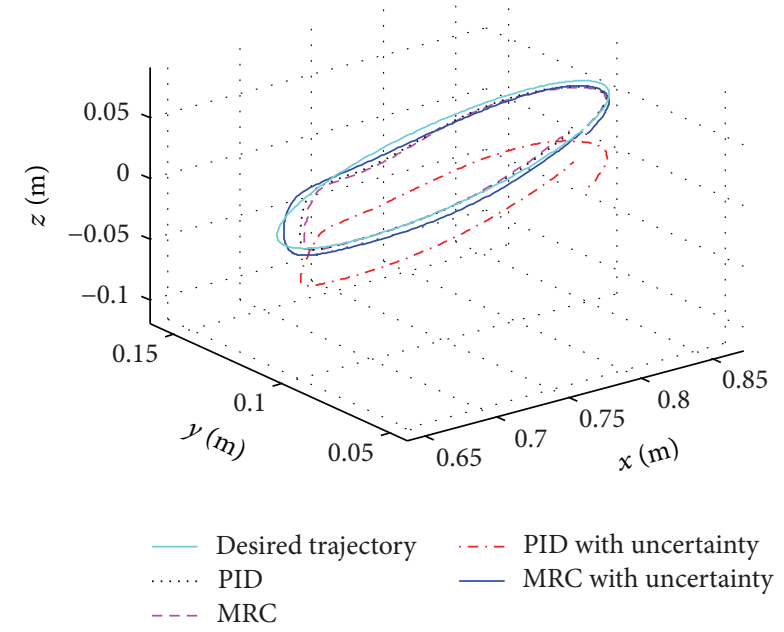

FIgURE 15: Task space $(x y z)$ trajectories (results from the circular trajectory).

(iii) an unknown ocean current is added in the simulation by properly considering the relative velocity of the in the dynamic model. It is assumed to have an irrotational current, constant in the inertial frame, and the value of the current is considered as $\boldsymbol{\nu}_{c}=$ $\left[\begin{array}{llllll}0.2 & 0.2 & 0.1 & 0 & 0 & 0\end{array}\right]^{T} \mathrm{~m} / \mathrm{s}$.

The results are presented in Figures 15-18, and from these results it is observed that the proposed controller is good in adapting the uncertainties and disturbances (refer to Figure 15). Figures 16 and 17 show that tracking errors of $x$ and $y$ in both PID and MRC methods are almost the same and results satisfactorily; however, the tracking error in $z$ substantiates that the proposed controller is comparatively good in the presence of uncertainties and disturbances.

4.3.4. Underwater Manipulator following a Given Desired Rectangular Trajectory with Vehicle Control. The rectangular trajectory chosen for the analysis has $0.4 \mathrm{~m}$ in width and

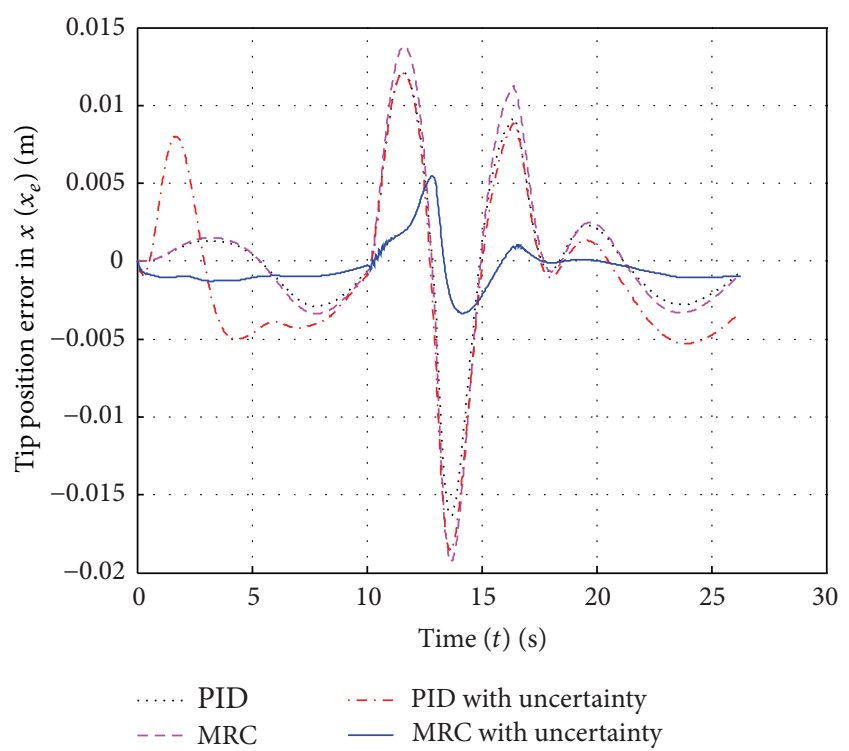

FIGURE 16: Time histories of the manipulator tip position tracking error in $x$ (circular trajectory).

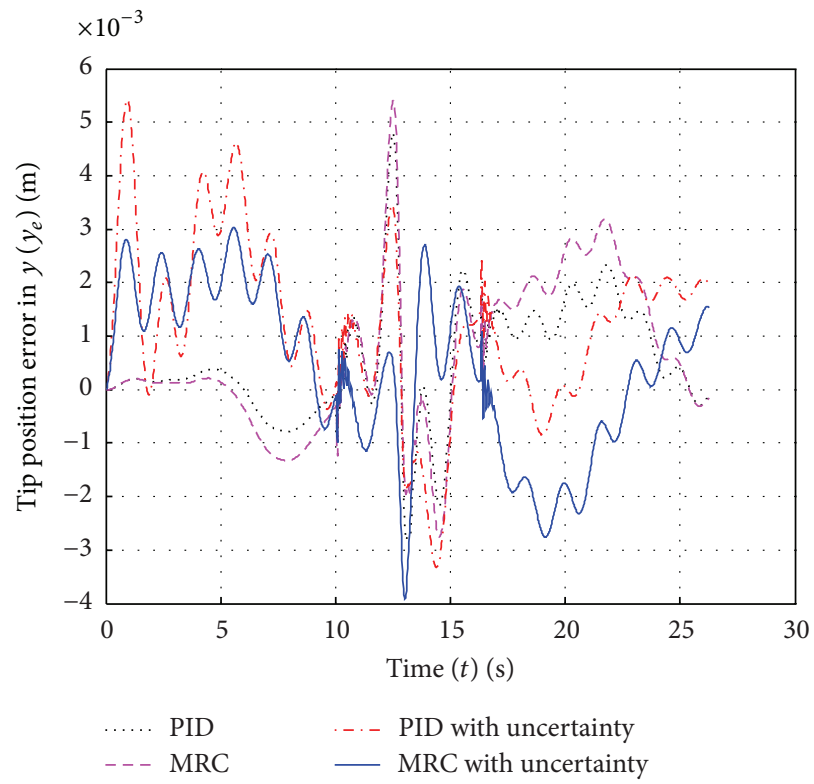

FIgURE 17: Time histories of the manipulator tip position tracking error in $y$ (circular trajectory).

$0.2 \mathrm{~m}$ in height in the $y z$ plane and an average speed of $0.05 \mathrm{~m} / \mathrm{s}$. As mentioned in the last case, the trajectory has been chosen with the consideration of the manipulator characteristics. Two conditions have been considered for the analysis: ideal condition (no disturbances, no uncertainties, etc.) and an uncertain condition (the same as the previous case Section 4.3.3). The results from computer simulations are plotted in Figure 19. In the ideal condition both controllers show quite satisfactory performance, and in the uncertain condition the proposed controller shows better performance. 


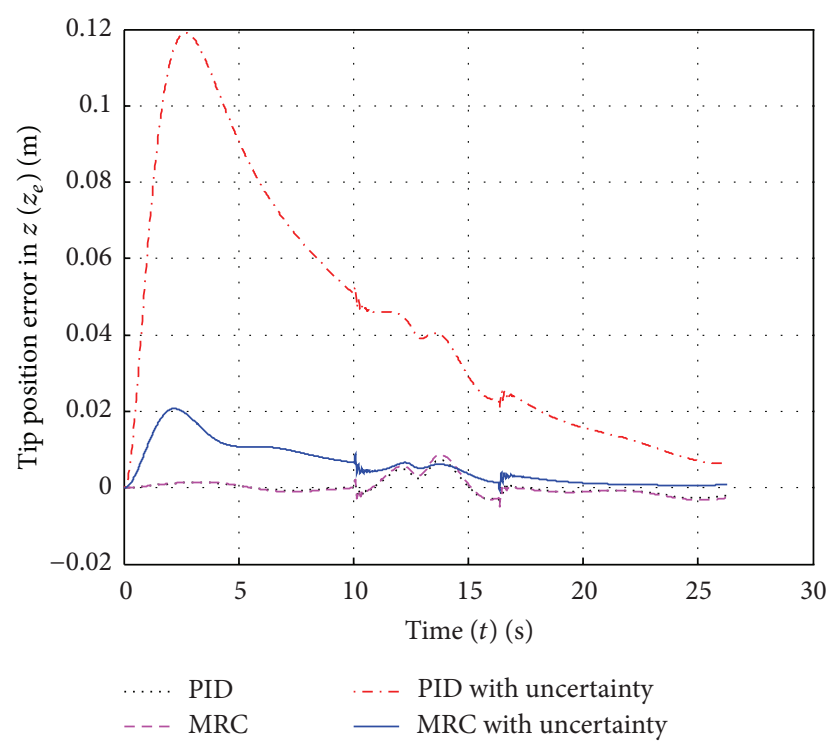

FIGURE 18: Time histories of the manipulator tip position tracking error in $z$ (circular trajectory).

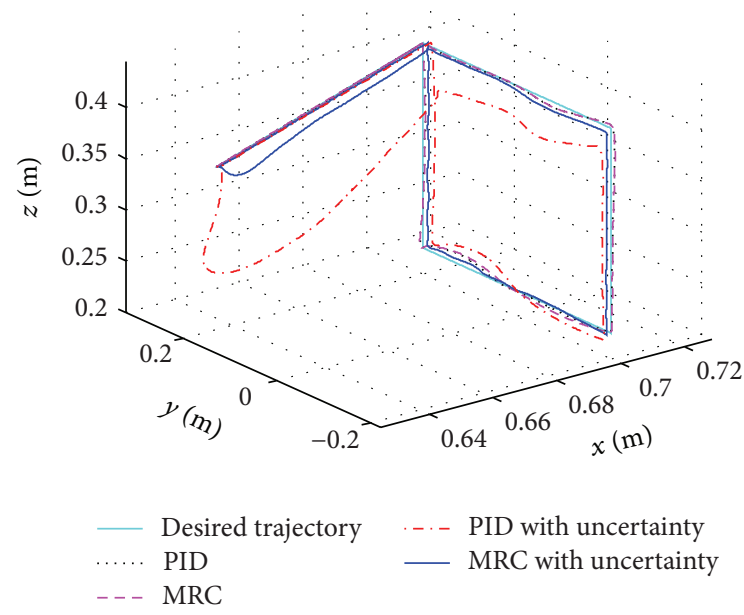

FIGURE 19: Task space $(x y z)$ trajectories (results from the rectangular trajectory).

In both trajectory tracking cases, the thruster forces do not exceed $\pm 5 \mathrm{~N}$, and the manipulator actuator torques stay within $\pm 0.15 \mathrm{Nm}$, which are well within the range of actuators.

\section{Conclusions}

In this study, the interaction effects due to the dynamic coupling between the vehicle and the manipulator were investigated using closed-form equations which provide a generalized scheme for formulating the equations of motion of the UVMS. The proposed scheme makes it possible to identify the structure, nature, and properties of the system, and it simplifies the control design. This study also proposed a model reference control approach for an underactuated
UVMS to perform underwater intervention tasks incorporating desired trajectory information. Extensive simulations were carried out to verify and demonstrate the effectiveness of the proposed scheme. In particular, the performance of the model reference controller is compared with that of the conventional PID controller for the given UVMS operation scenarios. The obtained results confirm the effectiveness and robustness of the proposed scheme in terms of tracking errors and control effort in the presence of external disturbances and parameter uncertainties. This research provides a generalized framework for modeling and controlling of an UVMS considering the dynamic coupling between the vehicle and the manipulator, which is crucial for achieving underwater manipulation tasks for a variety of scientific, industrial, and military missions using unmanned underwater vehicles.

\section{Conflict of Interests}

Authors confirm that this paper has not been published elsewhere and is not under consideration by another journal. All authors have approved the manuscript and agree with submission to Modelling and Simulation in Engineering. The authors have no conflict of interests to declare.

\section{Acknowledgments}

The author would like to acknowledge Professor Jinwhan Kim for his valuable suggestions and discussions on underwater manipulator. This research was supported in part by the World Class University (WCU) program through the National Research Foundation of Korea funded by the Ministry of Education, Science and Technology (R31-2008000-10045-0).

\section{References}

[1] G. Antonelli, F. Caccavale, S. Chiaverini, and L. Villani, “Tracking control for underwater vehicle-manipulator systems with velocity estimation," IEEE Journal of Oceanic Engineering, vol. 25, no. 3, pp. 399-413, 2000.

[2] G. Marani, S. K. Choi, and J. Yuh, "Underwater autonomous manipulation for intervention missions AUVs," Ocean Engineering, vol. 36, no. 1, pp. 15-23, 2009.

[3] S. McMillan, D. E. Orin, and R. B. McGhee, "Efficient dynamic simulation of an underwater vehicle with a robotic manipulator," IEEE Transactions on Systems, Man and Cybernetics, vol. 25, no. 8, pp. 1194-1206, 1995.

[4] T. I. Fossen, Guidance and Control of Ocean Vehicles, Wiley, Chichester, UK, 1994.

[5] T. J. Tarn, G. A. Shoults, and S. P. Yang, "A dynamic model of an underwater vehicle with a robotic manipulator using Kane's method," Autonomous Robots, vol. 3, no. 2-3, pp. 269-283, 1996.

[6] H. Mahesh, J. Yuh, and R. Lakshmi, "A Coordinated control of an underwater vehicle and robotic manipulator," Journal of Robotic Systems, vol. 8, no. 3, pp. 339-370, 1991.

[7] N. Sarkar and T. K. Podder, "Coordinated motion planning and control of autonomous underwater vehicle-manipulator systems subject to drag optimization," IEEE Journal of Oceanic Engineering, vol. 26, no. 2, pp. 228-239, 2001. 
[8] T. W. McLain, S. M. Rock, and M. J. Lee, "Experiments in the coordinated control of an underwater arm/vehicle system," Autonomous Robots, vol. 3, no. 2-3, pp. 213-232, 1996.

[9] M. W. Dunnigan and G. T. Russell, "Evaluation and reduction of the dynamic coupling between a manipulator and an underwater vehicle," IEEE Journal of Oceanic Engineering, vol. 23, no. 3, pp. 260-273, 1998.

[10] B. H. Jun, P. M. Lee, and J. Lee, "Manipulability analysis of underwater robotic arms on ROV and application to task-oriented joint configuration," in Proceedings of the IEEE OCEANS, pp. 1548-1553, November 2004.

[11] J. H. Ryu, D. S. Kwon, and P. M. Lee, "Control of underwater manipulators mounted on an ROV using base force information," in Proceedings of the IEEE International Conference on Robotics and Automation (ICRA '01), pp. 3238-3243, May 2001.

[12] M. Hildebrandt, L. Christensen, J. Kerdels, J. Albiez, and F. Kirchner, "Realtime motion compensation for ROV-based teleoperated underwater manipulators," in Proceedings of the IEEE OCEANS, pp. 1-6, May 2009.

[13] H. Shim, B. H. Jun, P. M. Lee, H. Baek, and J. Lee, "Workspace control system of underwater tele-operated manipulators on an ROV," Ocean Engineering, vol. 37, no. 11-12, pp. 1036-1047, 2010.

[14] J. J. Craig, Introduction to Robotics, Addison Wesley, Boston, Mass, USA, 1986.

[15] G. Antonelli, Underwater Robots: Motion and Force Control of Vehicle-Manipulator Systems, Springer, Berlin, Germany, 2006.

[16] K. Kim and T. Ura, "Applied model-based analysis and synthesis for the dynamics, guidance, and control of an autonomous undersea vehicle," Mathematical Problems in Engineering, vol. 2010, Article ID 149385, 23 pages, 2010.

[17] T. I. Fossen, Marine Control Systems: Guidance, Navigation and Control of Ships, Rigs and Underwater Vehicles, Marine Cybernetics AS, 2002.

[18] J. J. E. Slotine and W. Li, Applied Nonlinear Control, PrenticeHall, Upper Saddle River, NJ, USA, 1991.

[19] K. Y. Pettersen and O. Egeland, "Position and attitude control of an underactuated autonomous underwater vehicle," in Proceedings of the 1996 35th IEEE Conference on Decision and Control, pp. 987-991, Kobe, Japan, December 1996.

[20] T. H. Koh, M. W. S. Lau, G. Seet, and E. Low, "A control module scheme for an underactuated underwater robotic vehicle," Journal of Intelligent and Robotic Systems: Theory and Applications, vol. 46, no. 1, pp. 43-58, 2006.

[21] M. Santhakumar and T. Asokan, "Investigations on the hybrid tracking control of an underactuated autonomous underwater robot," Advanced Robotics, vol. 24, no. 11, pp. 1529-1556, 2010.

[22] ANSALDO Nucleare, Underwater Manipulator MARIS, 7080 Use and Maintenance Manual, Ansaldo Nuclear Division, Genoa, Italy, 1996.

[23] K. Deb, Optimization for Engineering Design: Algorithms and Examples, Prentice Hall, New Delhi, India, 2004. 

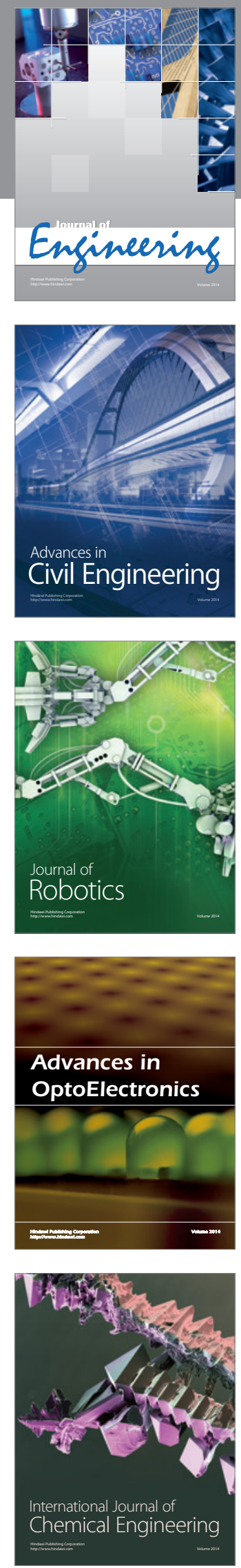

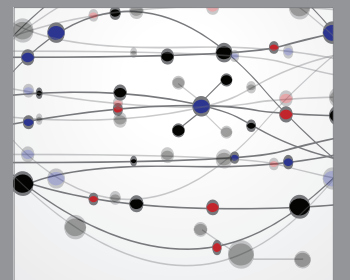

The Scientific World Journal
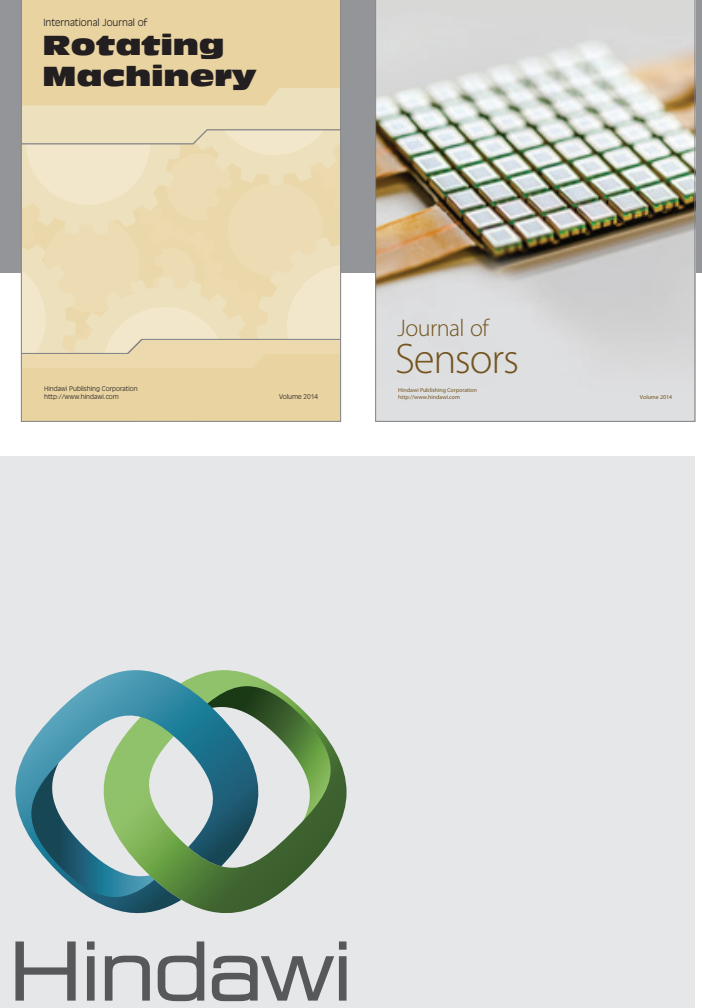

Submit your manuscripts at http://www.hindawi.com
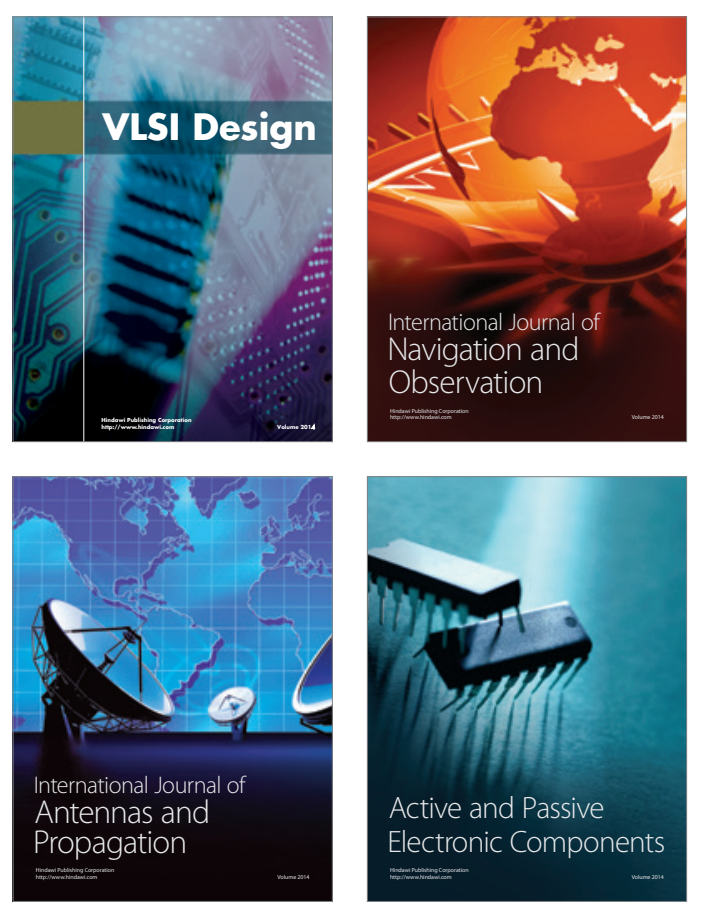
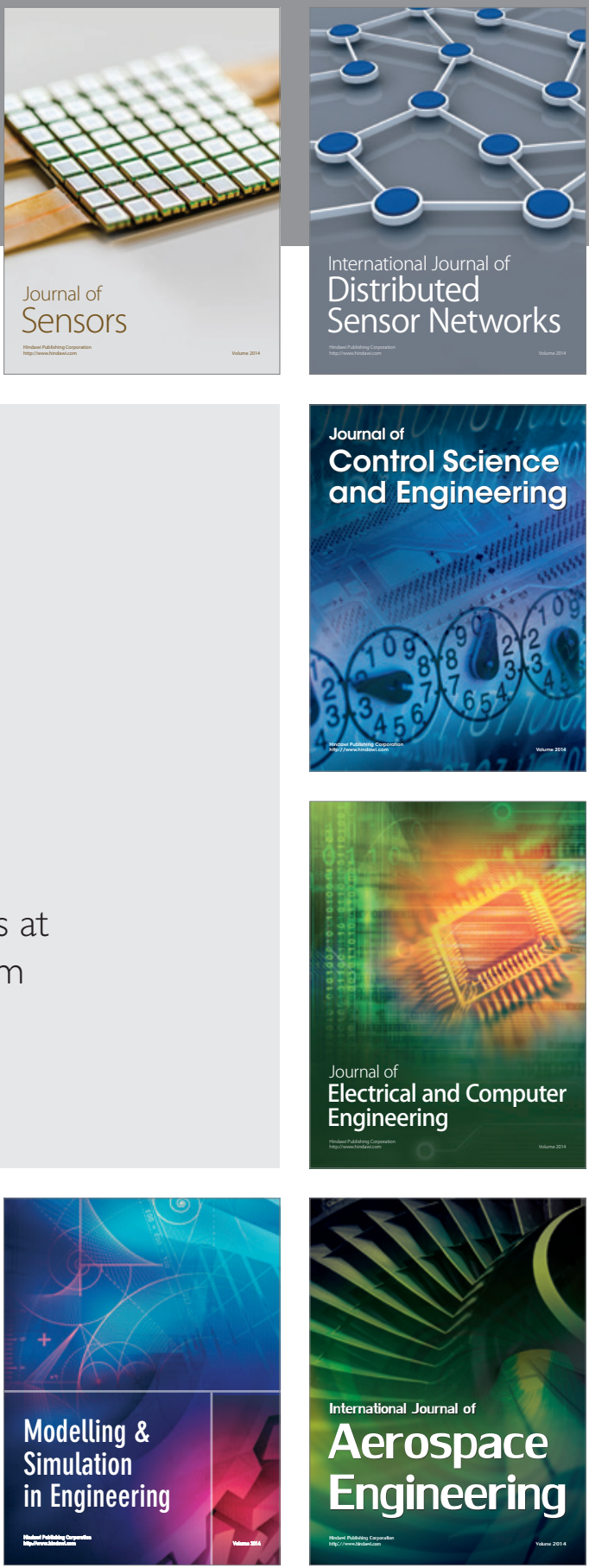

Journal of

Control Science

and Engineering
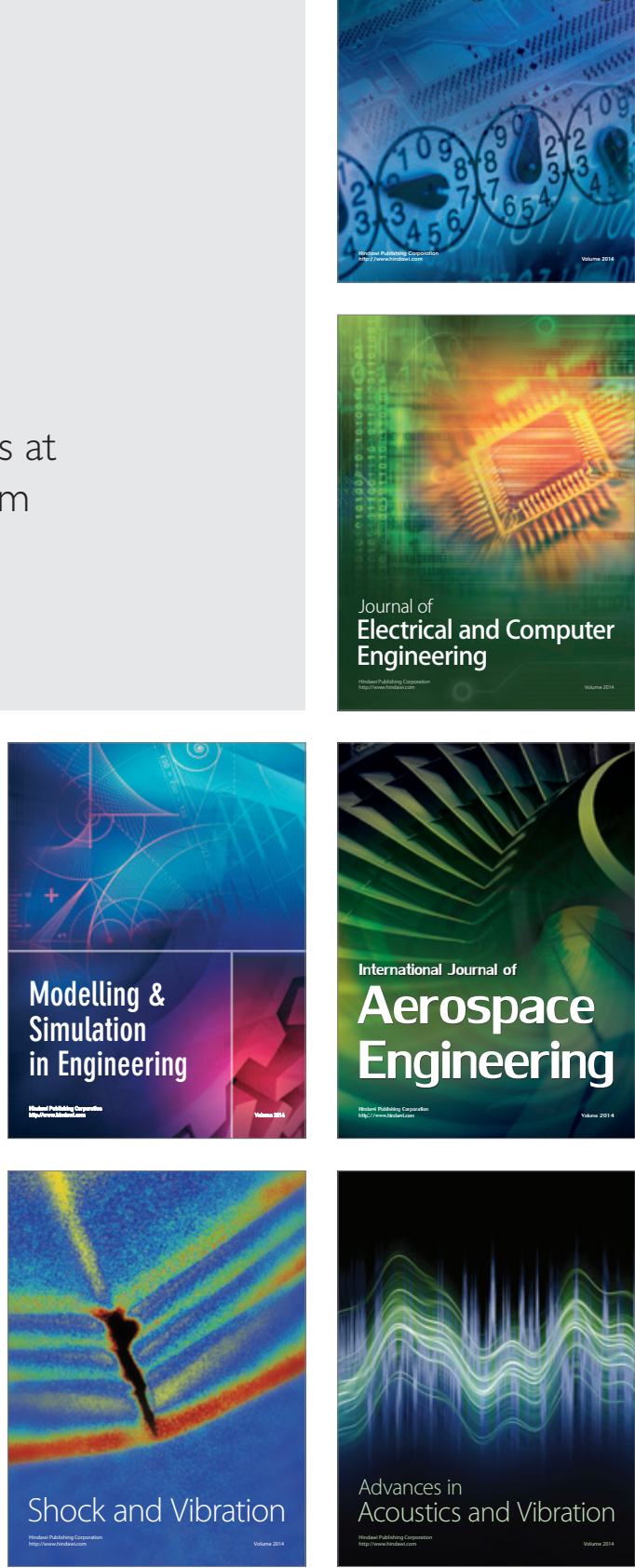